\title{
Fast Modelling of Field Evaporation in Atom Probe Tomography Using Level Set Methods
}

\author{
Charles Fletcher ${ }^{1}$, Michael P. Moody ${ }^{1}$, and Daniel Haley ${ }^{1}$ \\ ${ }^{1}$ Department of Materials, University of Oxford, Parks Road, \\ Oxford, OX13PH, United Kingdom
}

July 10, 2019

\begin{abstract}
Current imaging algorithms in Atom Probe Tomography (APT) assume a simple geometric model for the trajectories of evaporated ions between the emitter and detector. Such point-projection approaches fail to incorporate changes in sample geometry due to different phase evaporation behaviour, leading to severe distortions in reconstructed APT data. Here we propose a new approach to APT reconstruction where a continuum model is used to account for changes in sample geometry. A 2D model is demonstrated, readily extendable to $3 \mathrm{D}$, capable of simulating the complete evaporation of sample within minutes, while accounting for dielectric and crystallographic behaviour. Ion trajectories are calculated from the sample surface onto a simulated detector, allowing for the accurate mapping of detector positions onto the sample surface throughout time. Such an approach could drive a new reconstruction algorithm applicable to full size experimental datasets that can correct for the distortions currently present within APT reconstructions.
\end{abstract}

\section{Introduction}

Atom probe tomography (APT) is a destructive materials characterisation technique that combines a two-dimensional (2D) spatial detector with a mass spectrometer to analyse the atomic-level chemistry of tip shaped materials specimen. APT analyses rely on the physical phenomenon of field evaporation, where sample surface atoms, subjected to an intense electric field, are ionised and accelerated away from the tip onto a detector. Their exact trajectories depends on the sample geometry. As a result, traditional properties associated with imaging systems, such as the image magnification and Field-Of-View (FOV), begin to lose their applicability to describing such systems, as such properties can vary during the experiment. 
Subsequent to the experiment, a process known as reconstruction is performed whereby detected atoms' initial three-dimensional (3D) positions within the sample are estimated based off a number of assumptions. Current reconstruction algorithms are geometrically based, approximating ion trajectories via a simple mathematical projection from a spherical cap onto the detector, modelled as a plane [1]. During the reconstruction procedure experimentally detected ions are placed back onto this spherical cap based upon their detector coordinates, e.g. according to a stereographic or pseudostereographic projection $[1,2,3,4]$. By systematically shifting back the spherical cap after each sequential ion placement, a 3D point cloud is produced representing the initial sample positions of detected atomic species.

Atom probe's unique advantage is its ability to provide direct chemical information on a variety of different microstructures within complex material systems including quantum devices, transistors, and biomaterials $[5,6,7]$. However, the technique is not without its limitations, as complex physical effects occurring over various length scales introduce significant distortions of various origins into the reconstructions $[8,9,10]$. Without prior knowledge of the sample geometry, these distortions can be difficult to distinguish from true material microstructure. While point-projection reconstructions work well for homogeneous materials, its simplistic geometric projection assumptions break down within more complex material systems, where varying evaporation rates and dielectric behaviour across the sample can significantly impact the surface geometry and resultant ion trajectories. This is particularly true of semiconductor samples which typically exhibit multiple phases with varying evaporation fields and dielectric properties, introducing significant uncertainty into the parameter tuning of traditional projection-based reconstructions.

Alternative reconstruction protocols to the point-projection method have been proposed. One such approach by Beinke et. al. [11] proposes building up the sample atom-by-atom according to electrostatic simulation directly from the experimental hit-map data. However, such a method has stability issues arising from the current instruments' limited detection efficiency, the unstable nature of time-reversed growth problems [11], and the propogation of atom site displacement errors throughout the reconstruction arising from trajectory overlap. Such atomic-scale models are also restricted by the high computational and memory requirements of simulating full size experimental datasets, which can contain up to billions of atoms.

Another approach by Rolland et. al. [12, 13] proposes using an analytic geometric model, decoupled from the experimental data, that relaxes the hemispherical constraint to include multilayer samples by modelling the sample geometry as a stack of zero curvature surfaces. However, such a method remains constrained by geometric assumptions and approximates the field evaporation as a curvature driven effect. This greatly limits the range of cases to which it can be applied, and thus greatly limits its practical use. Additional modifications to the current point-projection method, where changes in the projection parameters are dynamically estimated throughout the reconstruction are highly empirical, and fail to correct for the local distortions arising at interfaces between phases 
of differing evaporation fields [14].

In this study we have developed a continuum model based off the level set method [15] for capturing changes in sample geometry due to electrostatically driven field evaporation. Such a model expands on previous work where the evaporation rate was approximated as mean curvature flow [16, 17], which we now move away from. A more detailed explanation on the relationship between mean curvature and electrostatics is given in [16]. The model has been implemented in Python, with program bottlenecks compiled through Cython (see Section 9 for source code). This extension retains the ability to simulate complete sample evaporation in only several minutes, while successfully capturing changes in phase evaporation field and surface faceting arising from underlying crystallographic anisotropy, previously not correctly modelled. Dielectric behaviour has also been incorporated through internal electrostatic simulation. By accurately modelling the evaporation of metals and semiconductors, this work provides a clear framework to drive new reconstruction algorithms capable of correcting many of the distortions seen in current reconstructions. Such a decoupled approach would also avoid the numerical stability issues typically arising in curvature driven time-reversed or growth problems $[11,18]$, relax the geometrical constraints of simple projection and specific curvature driven models, and maintain the computational efficiency required to perform reconstruction of full-size experimental datasets.

\section{The Atom Probe Reconstruction Problem}

During an experiment, surface atoms are ionised and removed from the sample under an applied electric field by a physical process known as field evaporation [19]. These surface ions are then accelerated away from the sample under the electric field and captured on a position-sensitive detector. Due to the electric field depending on the sample shape, the trajectories taken by evaporated ions will also be geometry dependent (see Figure 1). When the experiment is run in voltage mode, the electric field is applied in regular pulses, causing the sample to disintegrate and its geometry to change with time. The atom probe reconstruction problem is to estimate the spatial origins of detected atoms within the sample from the time-sequenced $2 \mathrm{D}$ detection data.

The critical component required to perform reconstruction is some sample specific function or mapping that describes how an ion at some particular detector coordinate and place in the sequence of detection events should be positioned within the constructed atom map (i.e. in 3D space) [20]. The exact trajectory taken by ions from sample to detector is unimportant in the context of performing the reconstruction, although these trajectories typically need to be estimated in order to obtain the start and end points.

The current point-projection based reconstruction algorithms avoid explicitly calculating trajectories by assuming they obey a pseudo-stereographic projection $[1,3,4]$. However, this approach makes an overly simplistic assumption about the sample geometry, which is known to govern the exact form 
of the external electric field and therefore ion trajectories. Tip shapes deviating from this hemispherical apex approximation have been observed experimentally via electron microscopy [21, 22].

Assuming an electrostatic approximation during each pulse, changes in sample geometry can be more accurately modelled through electrostaticallydriven simulation. As evaporated ion trajectories and the reconstruction function depend directly on the sample geometry evolution, such a modelling approach, when properly calibrated with experiment, would allow for an improved representation of the problem through reconstruction mappings correctly accounting for changes in sample geometry.

Our approach makes use of the boundary element method (BEM) [23] in order to solve the electrostatic field for a particular surface geometry. By coupling this electrostatic solver to the level set method for surface tracking, the evaporation of specific sample geometries can be modelled. In order to model dielectrics, the BEM is coupled to the finite element method (FEM-BEM) which solves the electric field over an internal mesh. Such an approach avoids having to construct an external mesh all the way from the specimen up to the detector. The BEM solves the electric field outside the sample via a boundary integral equation, allowing accurate calculation of ion trajectories onto the detector and the subsequent calculation of the reconstruction mapping. 


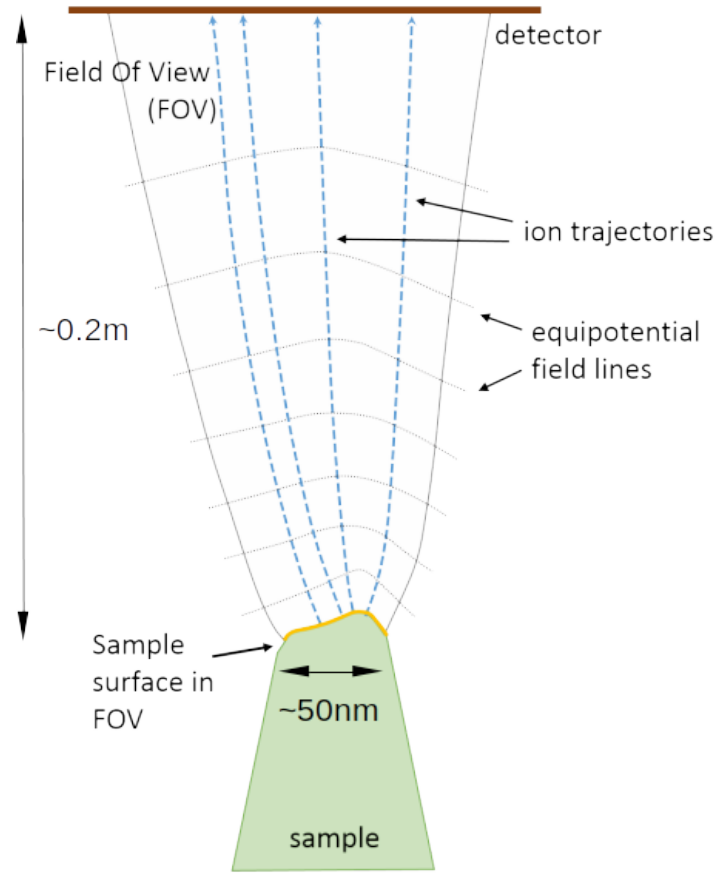

Figure 1: The setup for the atom probe reconstruction problem. Surface atoms are ionised by an applied electrostatic field and follow trajectories towards a 2D detector under this field. Note such a diagram is not to scale with a typical tip radius being $50 \mathrm{~nm}$ while trajectory flightpaths are approximately $200 \mathrm{~mm}$. 


\section{Theory}

\subsection{The Physics of Field Evaporation}

Within this work the local evaporation rate is taken to be an Arrhenius' law depending on the local electric field strength [24]. However, the framework proposed is highly flexible and allows for very general sample evaporation functions, not explored in the context of this work. The isotropic form of the assumed law is given by Eq. (1).

$$
\begin{aligned}
R_{e}(\mathbf{s}) & =A e^{-\beta\left(1-\frac{\|\mathbf{E}(\mathbf{s})\|}{F_{0}(\mathbf{s})}\right)} \\
F_{R} & =\max _{\mathbf{s} \in \mathbf{S}}\left(\frac{\|\mathbf{E}(\mathbf{s})\|}{F_{0}(\mathbf{s})}\right)
\end{aligned}
$$

Here $A$ is a global constant relating the field evaporation emission equation $[24,25]$ to the continuum theory, $\beta$ is a temperature dependent parameter, $F_{0}(\mathbf{s})$ is the local material evaporation field, $\|\mathbf{E}(\mathbf{s})\|$ is the local electric field strength, and $\mathbf{s}$ is a point on the sample surface $\mathbf{S}$. Whilst this law is of a simplified form to what is typically described within the literature [24], more complex formulations can be used without alterations to the model framework. Typical experimental values for $\beta$ approximately range from 40 (laser mode) to 400 (voltage mode). In this study $\beta$ has been assumed to be material independent. Throughout this study $\beta=40$ and field reduction $F_{R}=1.03$ (Eq. (2)).

\subsection{Level Set Interface Tracking}

The level set method is an approach to tracking the evolution of an $\mathrm{N}$ dimensional closed surface $\Gamma$ by embedding it within an scalar field $\phi$ in an $\mathrm{N}+1$ dimensional space $\boldsymbol{\Omega}$ (Eq. (3)).

$$
\begin{gathered}
\Gamma=\{\phi(\mathbf{x}) \mid \phi(\mathbf{x})=0\} \\
\phi(\mathbf{x})= \begin{cases}+d(\mathbf{x}, \Gamma) & \mathbf{x} \in \mathbf{\Omega}^{+} \\
-d(\mathbf{x}, \Gamma) & \mathbf{x} \in \mathbf{\Omega}^{-} \\
0 & \mathbf{x} \in \Gamma\end{cases} \\
\mathbf{n}(\mathbf{x})=-\nabla \phi(\mathbf{x})
\end{gathered}
$$

where $\mathbf{x} \in \boldsymbol{\Omega}, \Gamma \subset \boldsymbol{\Omega}, \boldsymbol{\Omega}^{+} \subset \boldsymbol{\Omega}$ is the subdomain inside the tracked surface, $\boldsymbol{\Omega}^{-} \subset \boldsymbol{\Omega}$ is the subdomain outside the tracked surface, and $d(\mathbf{x}, \Gamma)$ is the Euclidean distance between point $\mathbf{x}$ and the closest point on the tracked surface. As is typical for level set implementations [15], the initial form of $\phi(\mathbf{x})$ over $\boldsymbol{\Omega}$ has been taken to be the signed distance field (Eq. (4)). If $\phi(\mathbf{x})$ is given by Eq. (4), the outer surface normal $\mathbf{n}(\mathbf{s})$ at a surface point $\mathbf{s} \in \Gamma$ can 
then be obtained from Eq. (5), where $\|\ldots\|$ is the Euclidean norm. By evolving this scalar field according to the level set equation [15], the surface motion is implicitly tracked within the field. The equation of motion takes the form

$$
\frac{\partial \phi}{\partial t}+\nu(\mathbf{x})|\nabla \phi|=0
$$

where $\nu(\mathbf{x})$ is the velocity extension field [26], parallel to $\mathbf{n}(\mathbf{x})$ over $\boldsymbol{\Omega}$. This velocity extension must be defined on each iteration of the level set field and must converge to the correct physical value for the local velocity on the embedded surface as described by Eq. (7). In earlier studies, initial approaches for extension velocity construction simply extended the physics [26] such that the physical definition of the outer normal surface velocity (Eq. (1)) would be applied at each grid point using the locally calculated field value. However, with an electric field strength of zero inside any conducting material such a definition would be poorly suited to the sample evaporation problem. Recalculating the electric field for every grid point on each iteration would also be computationally expensive. A number of alternative methods exist for constructing such an extension velocity over every grid point $[27,28,29]$. For our current model we adopt the closest-point approach [27], largely due to its simplicity, where a grid point is assigned the velocity value of the closest point on the front. However, such a choice requires occasional reinitialization of $\phi(\mathbf{x})$ to restore its signed distance form. Future models could make use of a fast marching based velocity extension construction which could potentially avoid reinitialization [28, 29].

$$
\lim _{|\mathbf{x}-\mathbf{s}| \rightarrow 0} \nu(\mathbf{x})=R_{e}(\mathbf{s})
$$

where $R_{e}(\mathbf{s})$ is given by Eq. (1). The extension velocity construction allows for the physics of the tracked surface, in this case given by Eq. (1), to be coupled to the level set field. However, in order to calculate the evaporation rate the electrostatic solution over the sample surface must be calculated. Previous methods have involved finite elements [20,30] and finite differences [31, 32, 33]. However, such approaches require solving over some external mesh spanning the entire space over which ion trajectories will be integrated. Through BEM techniques, construction and calculation over external meshes can be avoided (see Section 3.3). The implicit representation of the tracked surface $\Gamma$ in the level set method avoids surface parameterisation issues arising in explicit contour tracking methods at corners [15] (critical when modelling faceted samples in APT). 


\subsection{The Electric Field Solution}

\subsubsection{The Atom Probe Electrostatic Problem}

The problem of calculating the sample surface electrostatic field in APT is described by Poisson's equation with zero free charges

$$
-\nabla(\epsilon(\mathbf{x}) \nabla u(\mathbf{x}))=0 \quad \forall x \in \mathbf{\Omega}
$$

where $\epsilon(\mathbf{x})$ is the local relative permittivity and $u(\mathbf{x}))$ is the electric potential such that $E(\mathbf{x}))=-\nabla u(\mathbf{x})$. Inside the sample $\epsilon(\mathbf{x})$ can vary depending on the local material properties whereas outside the sample $\epsilon(\mathbf{x})$ takes the vacuum permittivity value - simplifying to the Laplace Eq. (9). For purely conductive systems, the entire sample surface can be considered at the same potential as the base.

$$
-\nabla(\nabla u(\mathbf{x}))=0 \quad \forall x \in \mathbf{\Omega}^{-}
$$

Previous atom probe simulation approaches using level sets either employed a curvature approximation $[16,17]$ or a finite difference solution for the electric field [33]. The curvature approximation is a well understood problem and it is straightforward to define an extension velocity as a function of $\phi$. This velocity definition correctly converges at the tracked surface to its surface curvature, and is known to approximate the local electric field solution [16]. However, this approximation results in highly unphysical phenomena such as evaporation along the sample base and shank, the inability to form negative curvature (dimples) on surfaces, and requires decoupled electric field calculations in order to perform ion trajectory integration.

The finite difference solution of $\mathrm{Xu}$ et. al [33] represents a significant improvement through a direct coupling of the electric field solution to the level set model. However, it assumes the extension velocity to be a function of the local electric field. While strictly such a definition obeys Eq. (7), this choice causes $\phi$ to rapidly diverge from a signed distance form, particularly inside the sample where for conductors the electric field tends to zero. This causes the different level sets of $\phi$ to rapidly start "bunching up" at the sample surface, degrading the accuracy of the simulation [34]. To resolve this, frequent reinitialization of the level set field would be required, introducing further numerical drift in the sample surface [34]. A further computational issue with this approach, shared with other finite element approaches [30], is the requirement for a mesh that spans the entire simulation domain. This is particularly problematic when it comes to performing ion trajectory integration, where the sample size and detector distance differ by $\sim 10^{9}$, greatly increasing the computational demands. While adaptive meshes help to reduce this computational load [30], such an approach is not well suited to the level set framework where the mesh would have to be frequently rebuilt or modified as the sample geometry changes. Furthermore, it is possible to use alternate formulations to simplify and accelerate these electrostatic calculations, as described below. 


\subsubsection{The Boundary Element Method}

The Boundary element method (BEM) is a method for solving particular differential equations that can be arranged into an integral form around some boundary of known boundary conditions. Unlike alternative domain based methods, BEM does not require a solution at every point within the domain. An advantage of this integral form is its ability to determine the solution at specific locations within infinitely sized domains. This is particularly suitable to the atom probe problem, where the electric field only needs to be solved over the tip surface and at particular sites within a near infinite domain: along ion trajectories within the FOV.

Boundary integral based approaches have previously been used in APT. An atomic model by Rolland et. al. [35] solved the electrostatic field via a surface atom charge density through a discretization of the indirect boundary integral formulation [23]. BEM methods have also been used to investigate the dependence of the conventional point-projection reconstruction parameters with sample and instrument geometry [36]. However, this is the first time the method has been applied to fast continuum modelling of sample evaporation, while also accounting for sample dielectric behaviour.

The Laplace equation (9) is one such equation that can be reformulated into a boundary integral form for an infinite exterior domain [23]. By dividing the boundary into $k$ panels or boundary elements, the boundary integral can be represented as a sum of integrals over each boundary element. Discretising the boundary integral via a collocation method [23], whereby the equation is enforced at particular nodes on the boundary, allows analytical solutions for the boundary integrals to be obtained.

The simplest collocation BEM assumes a constant potential and normal flux on each panel. By imposing the discretized boundary integral equation at the central node on each panel, the collocation step, a system of $k$ linear equations can be constructed relating the boundary node potentials to normal fluxes. It is then straightforward to express this linear system as a matrix equation (10) [23].

$$
\mathbf{F} \cdot \mathbf{u}^{\text {bem }}=\mathbf{G} \cdot \mathbf{q}^{\text {bem }}
$$

where $\mathbf{F}$ and $\mathbf{G}$ are $k \times k$ problem matrices relating the $k$ surface potentials $\mathbf{u}^{\text {bem }}$ to the $k$ surface normal fluxes $\mathbf{q}^{\text {bem }}$ at boundary nodes. The problem matrices arise from discretisation of the boundary integral formulation [23], with the matrix elements of $\mathbf{F}$ defined in Eq. (19) and $\mathbf{G}$ defined in Eq. (20).

Finally, once the linear system given in Eq. (10) has been solved on the boundary, the electric field can be calculated at any point within the exterior domain via the discretized hypersingular boundary integral equation (11).

$$
\frac{\partial u(\mathbf{z})}{\partial x_{l}}=\sum_{j}^{k}\left[H\left(\mathbf{z}, \mathbf{x}_{j}\right) u_{j}-K\left(\mathbf{z}, \mathbf{x}_{j}\right) q_{j}\right]
$$

where $\mathbf{z} \in \mathbf{\Omega}^{-}, H\left(\mathbf{z}, \mathbf{x}_{j}\right)$ is defined in Eq. (14), $K\left(\mathbf{z}, \mathbf{x}_{j}\right)$ is defined in Eq. (15), $u_{j} \in \mathbf{u}^{\text {bem }}$, and $q_{j} \in \mathbf{q}^{\text {bem }}$. In two dimensions the electric potential 
solution drops away to negative infinity with increasing distance from the sample. However, in three dimensions the BEM framework offers the additional advantage in that the local electrode can be captured by fixing the system potential at infinity. The BEM framework also allows for the direct modelling of this local electrode through additional panels.

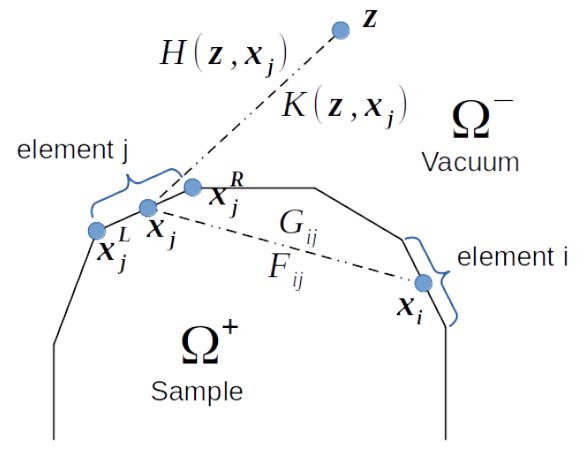

(a)

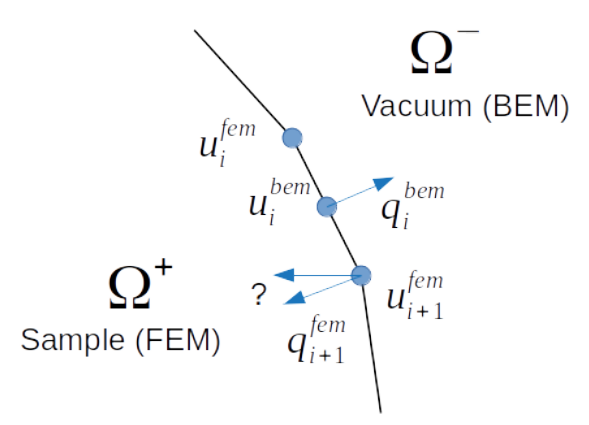

(b)

Figure 2: (a) Diagram showing the calculation of the matrix elements from the boundary coordinates. (a) Diagram showing the conformity of FEM and BEM nodes at the boundary. Note that nodes placed at element corners have ill-defined surface normals. Not to scale. 


\subsubsection{BEM Discretization}

Constant BEM matrix elements are constructed by calculating integral expressions associated with coordinate $\mathbf{z}$ and the boundary element $j$ [37] (see Figure 2(a)).

$$
\begin{gathered}
F\left(\mathbf{z}, \mathbf{x}_{j}\right)=-\frac{1}{2 \pi}\left(\arctan \left(\frac{s_{b}}{n}\right)-\arctan \left(\frac{s_{a}}{n}\right)\right) \\
G\left(\mathbf{z}, \mathbf{x}_{j}\right)=-\frac{1}{4 \pi}\left(s_{b}\left(\ln \left(n^{2}+s_{b}^{2}\right)-2\right)+2 n \arctan \left(\frac{s_{b}}{n}\right)\right. \\
\left.-s_{a}\left(\ln \left(n^{2}+s_{a}^{2}\right)-2\right)-2 n \arctan \left(\frac{s_{a}}{n}\right)\right) \\
H\left(\mathbf{z}, \mathbf{x}_{j}\right)=-\frac{1}{2 \pi}\left(\frac{s a}{n^{2}+s a^{2}}-\frac{s b}{n^{2}+s b^{2}}+\frac{n}{n^{2}+s b^{2}}-\frac{n}{n^{2}+s a^{2}}\right) \\
K\left(\mathbf{z}, \mathbf{x}_{j}\right)=-\frac{1}{2 \pi}\left(\arctan \left(\frac{s_{b}}{n}\right)-\arctan \left(\frac{s_{a}}{n}\right)-\frac{1}{2} \ln \left(n^{2}+s_{b}^{2}\right)+\frac{1}{2} \ln \left(n^{2}+s_{a}^{2}\right)\right)
\end{gathered}
$$

Here the scalars $n, s_{a}, s_{b}$ define a local coordinate dependent on the coordinate $\mathbf{z}$ and the boundary element $j$, on which the node $\mathbf{x}_{j}$ is centred. These local transformations are defined in Eq. (16), Eq. (17), and Eq. (18).

$$
\begin{aligned}
n & =\frac{1}{\left\|\mathbf{x}_{j}^{R}-\mathbf{x}_{j}^{L}\right\|}\left|\begin{array}{cc}
\left(\mathbf{z}-\mathbf{x}_{j}^{R}\right)_{x} & \left(\mathbf{z}-\mathbf{x}_{j}^{R}\right)_{y} \\
\left(\mathbf{x}_{j}^{L}-\mathbf{x}_{j}^{R}\right)_{x} & \left(\mathbf{x}_{j}^{L}-\mathbf{x}_{j}^{R}\right)_{y}
\end{array}\right| \\
s_{a} & =\frac{\left(\mathbf{z}-\mathbf{x}_{j}^{L}\right) \cdot\left(\mathbf{x}_{j}^{L}-\mathbf{x}_{j}^{R}\right)}{\left\|\mathbf{x}_{j}^{R}-\mathbf{x}_{j}^{L}\right\|} \\
s_{b} & =\frac{\left(\mathbf{z}-\mathbf{x}_{j}^{R}\right) \cdot\left(\mathbf{x}_{j}^{L}-\mathbf{x}_{j}^{R}\right)}{\left\|\mathbf{x}_{j}^{R}-\mathbf{x}_{j}^{L}\right\|}
\end{aligned}
$$

where $\mathbf{x}_{j}^{L}$ and $\mathbf{x}_{j}^{R}$ define the coordinate vectors of the left-hand and righthand corners of boundary element $j$, and $\|\ldots\|$ is the Euclidean norm. Finally, the matrix elements for the constructed BEM system on the boundary (Eq. (10)) are given by Eq. (19) and Eq. (20).

$$
\begin{aligned}
F_{i j} & =\left\{\begin{array}{cc}
F\left(\mathbf{x}_{i}, \mathbf{x}_{j}\right) & i \neq j \\
-\sum_{j \neq i}^{k} F\left(\mathbf{x}_{i}, \mathbf{x}_{j}\right)+1 & i=j
\end{array}\right. \\
G_{i j} & =G\left(\mathbf{x}_{i}, \mathbf{x}_{j}\right)
\end{aligned}
$$

where the coordinates $\mathbf{x}_{i}$ are the centre nodes of boundary elements. The relation for the diagonal terms of $\mathbf{F}$ is derived in [23]. 


\subsubsection{FEM-BEM Coupling}

While implementing a BEM model is sufficient for problems where the sample surface can be assumed to be equipotential (i.e. conductors), dielectric samples require the entire electric field to be solved both inside and outside the sample. In order to maintain the computational advantages of using the BEM to simulate the infinite exterior domain, a coupled scheme can be employed where the FEM solves the internal tip electrostatics while BEM solves the external electric field. Through this approach only an internal mesh needs to be rebuilt, which is limited in size, retaining the computational efficiencies for the much larger vacuum domain outside the sample.

Unlike the collocation BEM considered in Section 3.3.2, the finite element method is typically discretised from the weak form of the Poisson equation via a Galerkin method [38]. To obtain this formulation, the Eq. (8) is rearranged into the weak form by multipling by some arbitrary function and integrating over the domain giving

$$
\int_{\boldsymbol{\Omega}^{+}} \epsilon(\mathbf{x}) \nabla \nu(\mathbf{x}) \cdot \nabla u(\mathbf{x}) d \boldsymbol{\Omega}^{+}=\int_{\Gamma} \nu(\mathbf{x}) \epsilon(\mathbf{x}) \nabla u(\mathbf{x}) \cdot d \mathbf{\Gamma}
$$

where $\nu(\mathbf{x})$ is some arbitrary test function, $\boldsymbol{\Omega}^{+}$is the FEM problem domain (inside the sample), and $\Gamma$ is the FEM problem domain boundary (the sample surface). Proceeding with the Galerkin discretization [38], the domain is divided into triangle elements such that $M$ nodes are located at the FEM element corners. This triangle mesh is constructed such that the BEM elements conform with FEM element edges on the boundary (see Figure 2(b)). Using triangle basis functions centred on these FEM nodes, i.e. where basis function $\psi_{j}(\mathbf{x})$ is centred on $\mathbf{x}_{j}$, a linear system can be obtained

$$
\mathbf{A} \cdot \mathbf{u}^{f e m}=\mathbf{b}+\mathbf{N} \cdot \mathbf{q}^{f e m}
$$

where $\mathbf{A}$ is the problem's $M \times M$ stiffness matrix, $\mathbf{b}$ is the loading vector enforcing any Dirichlet boundary conditions (defined at a small patch of internal FEM nodes close to the sample base), $\mathbf{u}^{\text {fem }}$ is the FEM potential vector, $\mathbf{q}^{\text {fem }}$ is the expanded surface FEM normal flux vector (with zeros for internal FEM nodes not on the boundary), and $\mathbf{N}$ is some matrix capturing the Neumann boundary conditions contained in the right-hand side (RHS) of Eq. (21). The stiffness matrix construction for the potential problem is given in [38].

In order to solve the Poisson equation both inside and outside of the sample, the exterior BEM solution must be coupled to the FEM solution. The nodes of the constant BEM are located at the centre of the FEM element edges lying on the boundary. Therefore, in order to resolve this discrepancy an interpolating transfer matrix is required to express the BEM node values in terms of the FEM node values. This is achieved via averaging the potentials of the two FEM nodes located at each corner of the BEM element on the boundary. This potential averaging can be expressed as a matrix $\mathbf{T}^{f}$. The critical assumptions made in relating the BEM and FEM problems is given in Eq. (23), and the formulation of the FEM boundary integral in terms of $q_{j}^{b e m}$ is given in Eq. (24). 


$$
\mathbf{u}^{\text {bem }}=\mathbf{T}^{f} \cdot \mathbf{u}_{B}^{f e m}
$$

Here $\mathbf{u}_{B}^{f e m}$ is the reduced FEM potential vector consisting of FEM potential node values only on the boundary. Here $B$ is used to denote FEM nodes on the boundary while $\not{B}$ denotes nodes not on the boundary. Starting with the FEM system, the elements of the coupling matrix $N$ are obtained via Eq. (24) from the RHS of Eq. (21)

$$
\int_{\Gamma} \nu(\mathbf{x}) \epsilon(\mathbf{x}) \nabla u(\mathbf{x}) \cdot d \mathbf{\Gamma} \rightarrow-\sum_{j}^{M} q_{j}^{b e m} \int_{\Gamma} \psi_{i}(\mathbf{x}) d \Gamma
$$

The minus sign arises due to the boundary normals of the FEM problem being oriented inwards into its interior domain, compared to the boundary normals of the BEM problem, which are oriented outwards into its exterior domain. This allows for a coupled FEM-BEM system to be obtained

$$
\underbrace{\left(\left[\begin{array}{ll}
\mathbf{A}_{B^{\prime} B} & \mathbf{A}_{B, B} \\
\mathbf{A}_{B^{\prime} B} & \mathbf{A}_{B B}
\end{array}\right]+\left[\begin{array}{cc}
\mathbf{O} & \mathbf{O} \\
\mathbf{O} & \mathbf{N} \cdot \boldsymbol{G}^{-1} \cdot \boldsymbol{F} \cdot \mathbf{T}^{f}
\end{array}\right]\right)}_{\mathbf{A}^{\prime}} \cdot\left[\begin{array}{l}
\mathbf{u}_{B_{f}^{\prime}}^{f e m} \\
\mathbf{u}_{B}^{f e m}
\end{array}\right]=\left[\begin{array}{l}
\mathbf{b}_{B} \\
\mathbf{b}_{B}
\end{array}\right]
$$

Unlike $\mathbf{A}$, the effective stiffness matrix $\mathbf{A}^{\prime}$ cannot be rearranged into a symmetric form. Here $\boldsymbol{G}$ and $\boldsymbol{F}$ are the $k \times k$ BEM matrices from Eq. (10). The elements of the $k \times k$ coupling matrix $\mathbf{N}$ are given below

$$
N_{i j}= \begin{cases}\frac{1}{2}\left\|\mathbf{x}_{j}^{R}-\mathbf{x}_{j}^{L}\right\|, & \text { if } \mathbf{x}_{i} \text { and } \mathbf{x}_{j} \text { are adjacent boundary nodes } \\ 0, & \text { otherwise }\end{cases}
$$

Inverting the system matrix $\mathbf{A}^{\prime}$, the coupled FEM-BEM system given in Eq. (25) can be solved to give $\mathbf{u}^{\text {fem }}$. Following this the surface normal flux, centred on each mesh edge, can be obtained via Eq. (27)

$$
\mathbf{q}^{b e m}=\mathbf{G}^{-1} \cdot \mathbf{F} \cdot \mathbf{T}^{f} \cdot \mathbf{u}_{B}^{f e m}
$$

Finally, it should be noted that previous APT studies have found that relative permittivity under the electrostatic approximation fails to correctly model dielectric behaviour in APT due to high apex voltages and the laser [39, 40, 41, 42]. However, the wide applicability of FEM methods implies that with discretisation of the correct physics, fast and accurate modelling of dielectrics in APT could be possible via such a coupling framework. 


\subsection{Capturing Crystallographic Anisotropy}

While crystal anisotropy naturally arises in Voronoi cell-based atomic models for field evaporation through the mesh structure coinciding with the underlying physical Wigner-Seitz cells of the sample lattice [30, 43], incorporating this crystallographic structure into continuum models is not naturally inherent, and must be separately accounted for. While the level set model is at too low a resolution to account for surface atomic terracing, the model should be able to reproduce the faceting effects arising from the underlying sample lattice structure. Note that the exact resolution at which this continuum approximation breaksdown is beyond the scope of this study. Due to limitations in the current understanding of the basic physics involved behind field evaporation, the physically 'correct' way to incorporate this anisotropy into the model remains unclear. Even the approximate anisotropic curvature flow of the problem remains an active research area in the mathematics and crystal growth communities [44, 45, 46].

However, faceting behaviour can be reproduced by generalising the materialspecific evaporation fields to be a chosen anisotropic function. By specifying a set of parameters for particular material grains $\mathbb{F}=\left\{\gamma, \mathbf{f}_{0}, \mathbf{f}_{1}, \ldots, \mathbf{f}_{N}\right\}$, where $\mathbf{f}_{i}$ are oriented vectors corresponding to evaporation fields of particular fundamental facets (i.e. orientations related to the Miller indices), $N$ is the number of fundamental planes, and $\gamma$ is some isotropic constant, anisotropic evaporation can be included by generalising the material evaporation field (Eq. (28))

$$
\begin{aligned}
& F_{0}(\mathbf{s}, \theta)=\left\|\mathbf{f}_{\min }\right\|+\gamma\left|\tan \left(\theta_{\min }\right)\right| \\
& \tan \theta_{\min }(\mathbf{s})=\min _{\mathbb{F} /\{\gamma\}}\left\|\frac{\mathbf{n}(\mathbf{s}) \times \mathbf{f}_{i}}{\mathbf{n}(\mathbf{s}) \cdot \mathbf{f}_{i}}\right\| \\
& f_{\min }(\mathbf{s})=\underset{\mathbb{F} /\{\gamma\}}{\operatorname{argmin}}\|\underbrace{\frac{\mathbf{n}(\mathbf{s}) \times \mathbf{f}_{i}}{\mathbf{n}(\mathbf{s}) \cdot \mathbf{f}_{i}}}_{\tan (\alpha)}\|
\end{aligned}
$$

where $\mathbf{n}(\mathbf{s})$ is the local surface outer normal given by Eq. (5), and $\mathbb{F}$ the set of parameters associated with the local material grain. Note that the exact form of the anisotropy function only effects the transition behaviour of the sample geometry and not its final faceted equilibrium form [47] (that being determined by the magnitude and angular positions of the anisotropy function minima). These anisotropy functions can be visualised as a function of angle $\alpha$ for particular crystal grains (an example will be shown later in Figure 15(a)). Currently this anisotropy has been incorporated into the model by generalising the evaporation field parameter $F_{0}$.

This physical parameter is known to play at least some role in facet formation due to its dependence on the work function and surface bond configuration, both

of which vary with surface crystallography [24]. However, atomic models have 
shown that sample faceting also arises from electrostatics on a crystallographic specimen regardless of evaporation fields, implying that perhaps $F_{0}$ is not the only cause [48].

Anisotropic effects could also be included through alternative parameters within the evaporation law (1), i.e. by making $A$ material dependent. The exact form of the anisotropic function used in this study, defined in Eq. (28), has largely been assumed, although its form has been guessed at by assuming an approximate linear relation between the evaporation field and the surface free energy [49]. A more physical form for the faceting function, capturing the true transient behaviour between steady states of the emitter, would have to be derived via more fundamental simulation or experiment. 


\section{Simulation Method}

The basic algorithm for modelling sample evaporation consists of a level set method for tracking the sample surface combined with an electrostatic simulation, either BEM or FEM-BEM, for calculating the surface electric field strength and ion trajectories. The surface field strength gives the sample surface evaporation rate through the assumed evaporation law (1) which is used to construct an extension velocity for coupling to the level set field via a closestpoint approach [27]. The major steps in this algorithm are outlined in Figure 3. The outlined framework could be readily extended to $3 \mathrm{D}$ without significant modification.

\subsection{Model Initialisation}

The sample geometry is initialised via an input image, with different phases or grains represented by different colours. By using an image resolution greater than that of the level set grid resolution, a subgrid positioning of the sample surface can be achieved on initialisation. The evaporation parameters associated with the different phases are looked up within a database according to a unique identifier. The initialisation also consists of a rotation parameter associated with each grain (defined by a single angle in 2D), performed on the oriented facet vectors $\mathbf{f}_{i}$, in order to capture misorientation. Through this initialisation procedure evaporation parameters are associated with all level set grid cells, and from this the evaporation parameters of each surface panel are determined by which cell panel centres fall.

In this study a level set grid resolution of $128 \times 128$ has been used, which for the sample geometries simulated results in boundary elements of around $0.5 \mathrm{~nm}$, roughly in line with the lateral resolution of homogeneous samples within APT [50]. APT simulations define 1 grid cell to be $1.0 \mathrm{~nm}$ in this study. A virtual detector was positioned $0.2 \mathrm{~m}$ from the sample.

\subsection{Integrating the Level Set Field}

The level set equation of motion (6) has been discretized via a finite difference method on a fixed Cartesian grid. During each iteration of the level set field, the extension velocity $v(\mathbf{x})$ is constructed from the sample surface calculated values according to Eq. (1) via a closest point approach [27] and a dynamic timestep determined according to the Courant-Friedrichs-Lewy (CFL) condition. Time integration is performed via either Euler's method or Heun's method using a first-order upwind scheme. The sample surface contour is extracted from the level set field via the Marching Squares algorithm [51]. 


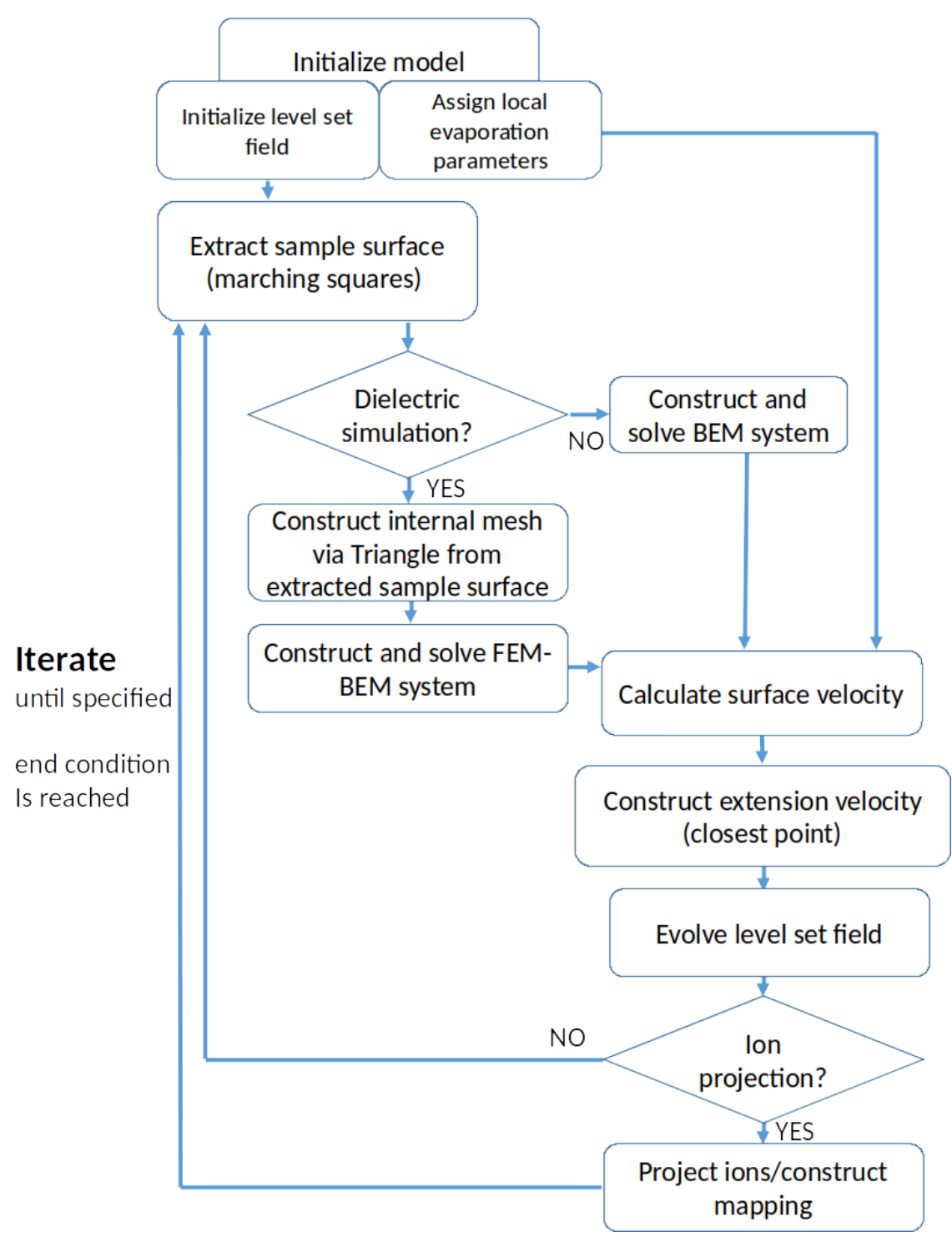

Figure 3: Flowchart showing major components of the simulation algorithm presented in this study. 


\subsection{Solving the Electric Field}

In order to calculate the sample surface evaporation rate, the electric field strength over the surface must be calculated via BEM or FEM-BEM, depending on whether dielectric effects are to be included. The matrix elements of the BEM and FEM-BEM were calculated analytically via compiled Cython, according to the methods outlined in Sections 3.3.3 and 3.3.4. During each calculation of the electric field, a user-defined fraction of the simulated tip is used to estimate a shank angle (see Figure 4(a)). The tip is then extended by an additional number of user-defined boundary elements outside of the level set grid domain, as shown in Figures 4(b). Such an approach requires only the evaporating apex of the sample to be tracked via the level set method, with the shank geometry being appended separately during field calculations. For the FEM-BEM coupling, the internal FEM mesh was constructed using the mesh generation program Triangle [52]. The FEM mesh was constructed with element edges being enforced on the sample surface and on internal interfaces between different material phases. Currently both the BEM and the coupled FEM-BEM linear system are solved directly via LU decomposition (in this implementation the FEM-BEM linear system is solved using the SciPy sparse matrix package). The model setup for the BEM and FEM-BEM approaches are shown in Figures 4(b) and 4(c). Further information regarding implementation can be found through the source code comments and repository documentation (see Section 9).

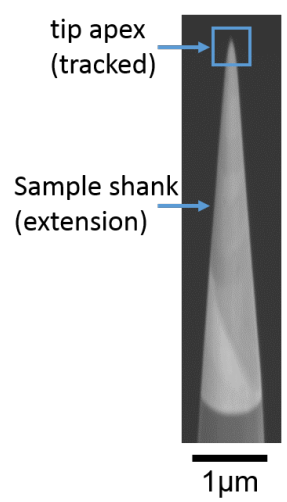

(a)

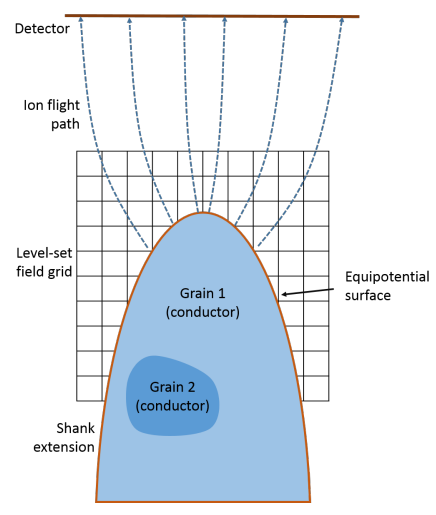

(b)

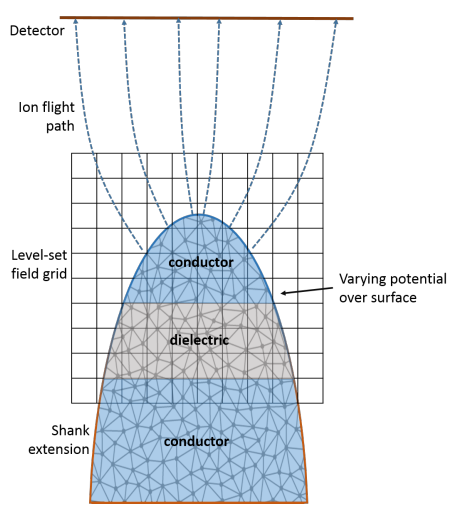

(c)

Figure 4: (a) An example SEM image of a typical steel sample used in APT. (b) BEM simulation setup for simulating conducting materials. (c) Simulation setup for FEM-BEM extension to dielectrics. The irregular FEM mesh allows the FEM and BEM problem boundaries to coincide. Schematics not to scale. 


\subsection{Reconstruction Mappings}

In order to perform atom probe reconstruction, some time-dependent function determined by the sample geometry is required that estimates the initial positions of detected ions prior to evaporation. Currently this function must be a bijection, providing a coordinate in $3 \mathrm{D}$ space for each detector position. It must also span the entire FOV, such that any detected ion can be uniquely positioned. It is this reconstruction mapping that contains the positioning information required to perform atom probe reconstruction.

In order to build up the reconstruction mapping, ion projection can be performed at distributed points over the sample surface using an adaptive-step fifth-order Runge-Kutta (RK5) integrator [53]. In this study ion trajectories were launched at the corners of each boundary element above a certain fraction of the tracked sample apex height (taken as the top $15 \%$ of the sample apex), a trajectory sampling in line with APT lateral resolution [50]. Ions were launched at boundary element corners some set initial distance normal to the sample surface (taken to be $0.1 \mathrm{~nm}$ ). This projection step can be performed at different times throughout the simulated tip evaporation after a sufficient fraction of sample area had been evaporated. Trajectory integration is performed up to the simulated detector, located $0.2 \mathrm{~m}$ from the sample, where the final detector positions of these sampled ions are recorded. Using these sampled trajectories, the corresponding position on the sample surface for an arbitrary point on the detector can then be estimated through linear interpolation (see Figure 5). Currently this method for constructing the mapping fails to account for bijective breakdown arising from trajectory overlap [54].

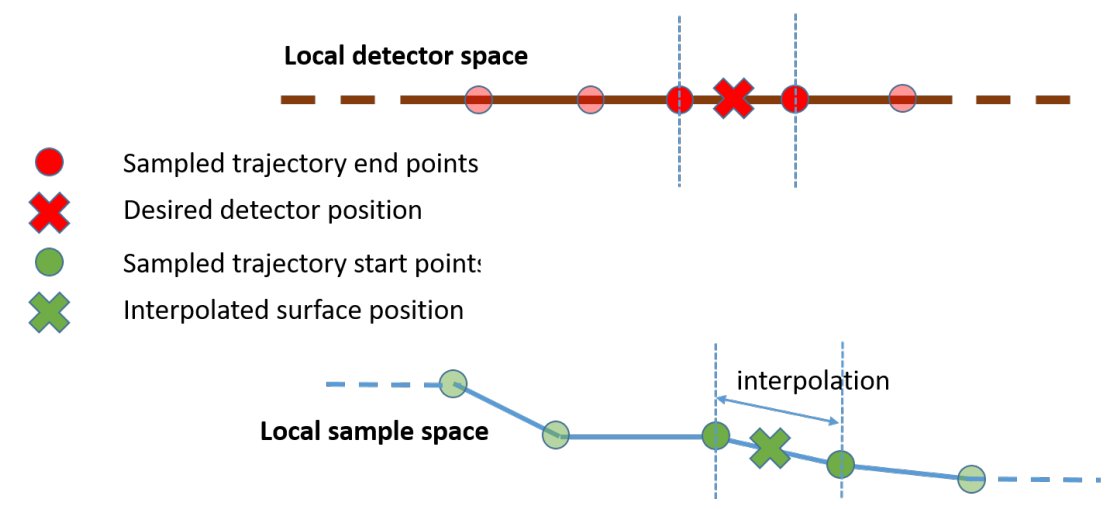

Figure 5: Diagram showing the procedure behind the construction of the reconstruction mapping. Note that while a linear interpolation scheme has been shown, higher order surface interpolation schemes are also possible. 


\section{Results and Discussion}

\section{$5.1 \quad$ Numerical Stability}

In order to check the correctness and numerical accuracy of the level set method, a simple collapse test can be performed in which a sphere is collapsed according to a particular velocity function. An analyical solution can be obtained for the collapse time due to the problem's symmetry. Initially, this collapse test was performed using a linear velocity law (31).

$$
\nu(\mathbf{s})=\frac{d R}{d t}=-B \frac{E(\mathbf{s})}{F_{0}}
$$

where $R$ is the circle radius as a function of time, $\mathbf{s}$ is a point on the surface, $B$ is a global constant, $E$ is the electric field calculated via BEM, and $F_{0}$ is an isotropic material evaporation field. The expression for the surface electric field of a sphere with constant charge $Q$ is given by $E=\frac{Q}{2 \pi R}$. This is similar in principle to the mean curvature flow case [17], but not identical, as here the tip is being held at a constant voltage $u_{c}$, where $u_{c}=\frac{Q}{2 \pi} \ln (R)$, rather than at a constant charge (equivalent to the curvature collapse case). Therefore, substituting out $Q$, the actual differential equation governing the change in radius is given by

$$
\frac{d R}{d t}=-\frac{B u_{c}}{F_{0}} \frac{1}{R \ln (R)}
$$

A similar expression can be constructed for the collapse of a fixed potential circle geometry under an Arrhenius law (1). This is given by

$$
\frac{d R}{d t}=-A e^{-\frac{\beta}{2}\left(1-\frac{1}{F_{0}} \frac{1}{R \ln (R)}\right)}
$$

Finally, by plotting the level set solutions for collapse under these two laws against the numerical solutions obtained from Eq. (32) and Eq. (33), the accuracy of the level set solver can be gauged. The test shows a slightly faster collapse for the level set obtained solution in the Arrhenius case (Figure 6). However the shape is generally well preserved, and the simulation resolution is low. While this divergence was present for both Euler's and Heun's time integration, it reduced with decreasing timestep. 


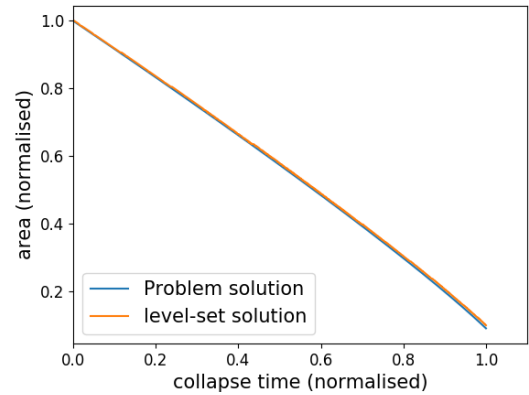

(a)

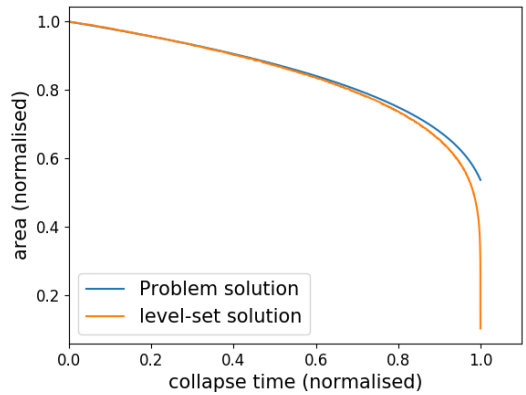

(b)

Figure 6: Level set solution in comparison to the true solution for circle collapse problem using an Euler integrator. (a) Linear law collapse, as in Eq. (32). (b) Arrhenius law collapse, as in Eq. (33). Collapse was defined to have occured at $5 \%$ of the initial sample area.

When using the Arrhenius' law the major source of error arises from the constant BEM approximation, where even small errors in the calculated surface flux value are amplified under the exponential law. This error was present for both Euler's and Heun's time integrators, and could only be reduced by using a sufficiently small timestep. In this study the timestep is chosen dynamically as a fraction $\delta$ of the Courant-Friedrichs-Lewy (CFL) condition $d t=\delta \frac{h}{\max _{\mathbf{x} \in \boldsymbol{\Omega}}|\nu(\mathbf{x})|}$, where $h=1 \mathrm{~nm}$ and $|\nu(\mathbf{x})|$ is the absolute value of the velocity extension field. The result of this error is a 'chipping effect' into the surface that becomes present at sufficiently large time intervals, resulting in divergence of the tracked geometry from a circle (Figure 7). Such errors could be reduced by implementing higher-order BEM methods $[55,56]$ or switching over to a Galerkin based method [57, 58]. For the purposes of this work however, the results show that the implementation is correctly following the analytical model, and that the accuracy can be improved with a higher computational cost during the level set integration. This is sufficient to model tip evaporation with higher accuracy and correctness than our prior APT level set works [16, 17]. All following simulations use a CFL fraction of $\delta=0.1$ and reinitialize the level set field every 50 iterations. This was found to prevent significant drift from the problem solution (see Figure 6) and minimise detector isoline instabilities (see Figure 8(c)). 


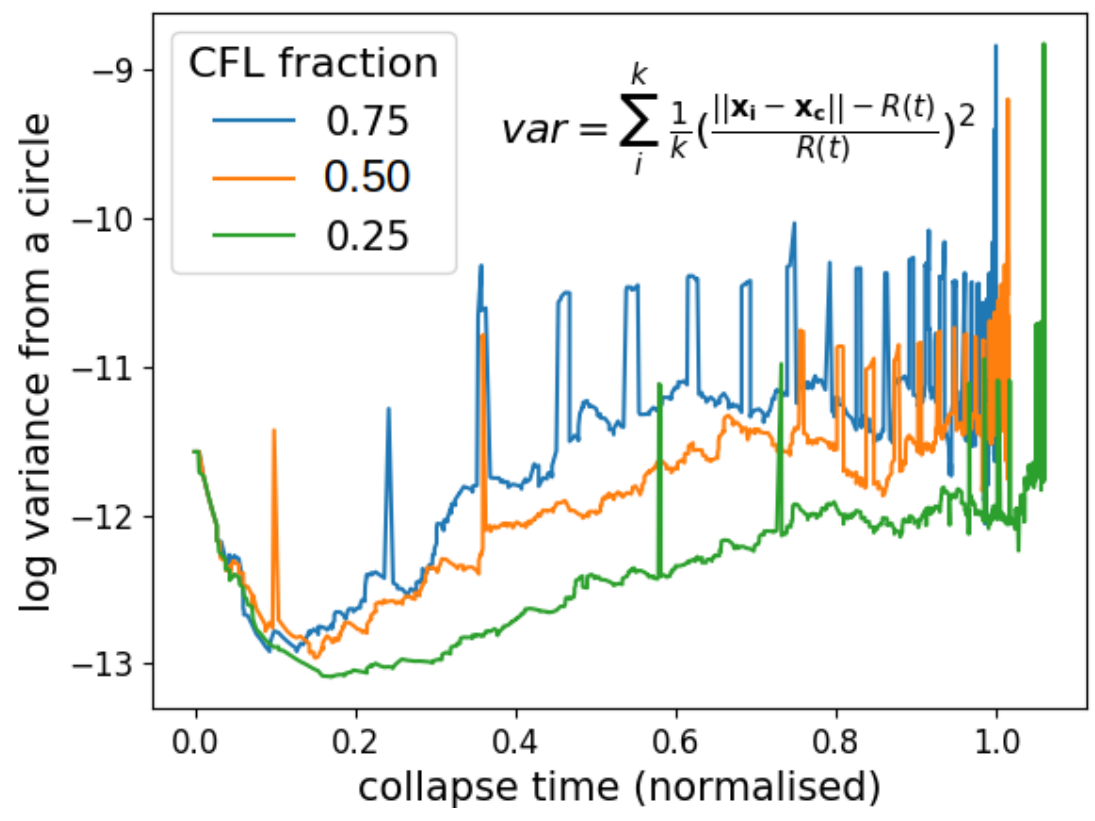

Figure 7: Log variance from perfect circle against different CFL timestep fractions for Arrhenius' driven collapse. Some numerical issues still exist (spikes in variance), although these are small enough not to be of concern in this study. In the variance formula $\mathbf{x}_{\mathbf{i}}$ are the boundary element centres, $\mathbf{x}_{\mathbf{c}}$ is the circle centre, and $R(t)$ is the circle radius calculated from its instantaneous area. 


\subsection{Multilayers and Precipitates}

First the simpler pure BEM configuration is considered (see Figure 4(a)) with anisotropy disabled. This corresponds to a model for isotropic conductors where each phase has an individual evaporation field $F_{0}$. The surface electric field can be calculated via constant BEM (10), assuming the boundary elements to be equipotential.

Figure 8 shows a simulation for a sample geometry containing a lower evaporation field multilayer (coloured red with an evaporation fields of $30 \mathrm{Vnm}^{-1}$ and $20 \mathrm{Vnm}^{-1}$ ). The Figures $8(\mathrm{a})$ and $8(\mathrm{~b})$ show a frame of the simulated sample evaporation, along with ion projection from the surface and the corresponding one-dimensional (1D) simulated hitmap. Note that prior to evaporation the initial geometry of the sample apex was hemispherical. The lower evaporation field of the multilayer results in a local reduction in positive surface curvature, causing a focusing of ion trajectories and a subsequent increase in the projected hitmap density.

Following a period of evaporation, the reconstruction mapping can be constructed and visualised through the plotting of detector isolines corresponding to how the origins of evaporated surface ions arriving at fixed detector positions change throughout the experiment (Figure 8(c)). The initial increase in isoline density corresponds to a period of trajectory magnification just prior to reaching the multilayer. On evaporation into the multilayer the isoline density decreases and spacing increases, corresponding to reduced magnification resulting from a blunting of the apex. The mapping also directly reveals the narrowing and subsequent widening of the FOV on passing through the multilayer.

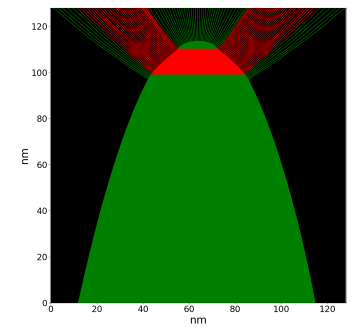

(a)

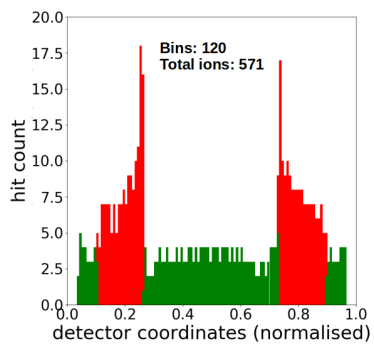

(b)

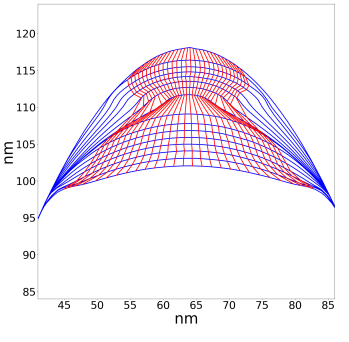

(c)

Figure 8: Simulation of a lower evaporation field multilayer ( $F_{0}$ values of $30 \mathrm{Vnm}^{-1}$ and $20 \mathrm{Vnm}^{-1}$ ). (a) Partial evaporation of multilayer with ion projection. (b) Corresponding simulated 1D hitmap. (c) Reconstruction mapping for multilayer structure where blue lines represent the sample surface at different times while red lines are the detector isolines described above. Note that electrostatics were solved in $2 \mathrm{D}$ and so predicted lensing behaviour cannot be directly compared to $3 \mathrm{D}$ models or experiment. 
Another specimen geometry of interest is that of precipitates, as shown in Figure 9(a). The Figures $9(\mathrm{~b})$ and 9 (c) contain the reconstruction mappings for a high-field $\left(F_{0}\right.$ values of $30 \mathrm{Vnm}^{-1}$ and $\left.35 \mathrm{Vnm}^{-1}\right)$ and low-field $\left(30 \mathrm{Vnm}^{-1}\right.$ and $20 \mathrm{Vnm}^{-1}$ ) precipitate. In both cases the changing geometry during the precipitate's evaporation results in a temporary change in magnification. The high-field precipitate reveals an increase in magnification over the precipitate followed by a region of demagnification over the rest of the sample surface in the FOV. This trajectory focusing is greatest at the material interface between the precipitate and bulk, as seen by where the isoline spacing is at its greatest. The low-field precipitate reveals a decrease in magnification over the precipitate, as seen by the increased isoline spacing. Sites of extreme focusing and overlap of trajectories are observed as spatial instabilities in individual isolines. Note that in both cases the local microstructure of the precipitate influences ion trajectories over the entire FOV. This analysis reveals that such structures cannot be assumed to introduce only local distortions into reconstructions.

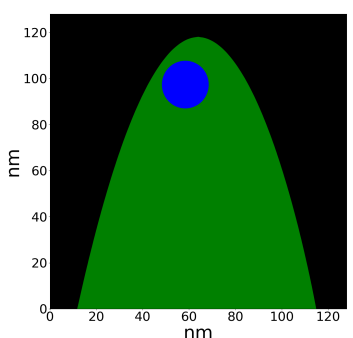

(a)

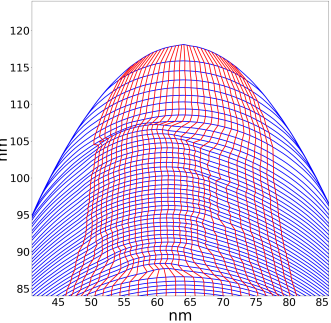

(b)

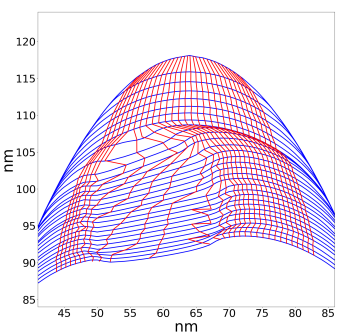

(c)

Figure 9: (a) Initial simulated geometry containing an off-axis precipitate. (b) Reconstruction mapping for a high-field precipitate $\left(F_{0}\right.$ values of $30 \mathrm{Vnm}^{-1}$ and $\left.35 \mathrm{Vnm}^{-1}\right)$. (c) Reconstruction mapping for a low-field precipitate $\left(F_{0}\right.$ values of $30 \mathrm{Vnm}^{-1}$ and $20 \mathrm{Vnm}^{-1}$ ). Blue lines represent the tip surface at different times while red lines represent the detector isolines. Note that electrostatics were solved in 2D and so predicted lensing behaviour cannot be directly compared to $3 \mathrm{D}$ models or experiment. 


\subsection{The FinFET Geometry}

An active area of research in APT where reconstruction distortions are particularly problematic is that of imaging and analysing semiconductor devices $[59,60,6,61]$. Such materials systems typically consist of phases of varying evaporation fields and dielectric properties which can result in the trajectory mapping significantly diverging from that assumed by the traditional point-projection method.

While previous studies have modeled the evaporation of typical semiconductor geometries $[62,10]$, it is still beneficial to compare with the results produced by the continuum model. In the study by Melkonyan et. al. [6], a sample representing a finFET-like structure was analysed and distortions compared to those produced by a finite difference model [31]. Reconstructing the same dataset via point-projection (Figure 10(a)) for a constant shank angle [3] reproduces the same 'narrowing' distortion of the central fin as observed in the original analysis.

This narrowing artifact of the central fin can be explained purely in terms of a changing sample geometry due to a different evaporation behaviour of the central fin compared to the surrounding material . By assuming a lower evaporation field for the central fin of $20 \mathrm{Vnm}^{-1}$ compared to the surrounding material's $30 \mathrm{Vnm}^{-1}$, the resulting change in apex geometry brings about an increased focusing of ion trajectories originating from this central fin phase. This trajectory focusing shows up as an increased hit density region on the simulated 1D hitmap (Figure 10(c)) which narrows and increases in density with further evaporation of the sample (Figure 10(e)). Under the simple geometric assumptions of the point-projection algorithm this trajectory focusing and increased hit density remains uncorrected for, resulting in the narrowing of the central fin within the reconstruction.

Once again the reconstruction mapping provides a useful method for comparing the local magnification effects during sample evaporation (see Figure 10(f)). The increasing trajectory focusing arising from the central fin can be understood by the increasing spacing between detector isolines within the centre of the mapping. At the interface between the central fin phase and surrounding material there is a region of enhanced trajectory focusing and overlap, characterized by spatial instabilities in the isolines, followed by a region of high magnification where the density of detector isolines increases. Interestingly, the mapping predicts there to be a sudden increase in the FOV roughly half way into the simulated evaporation. 


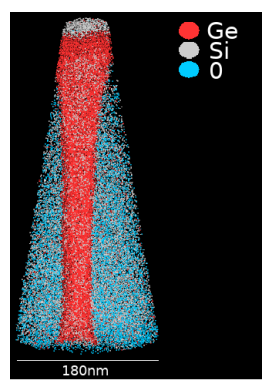

(a)

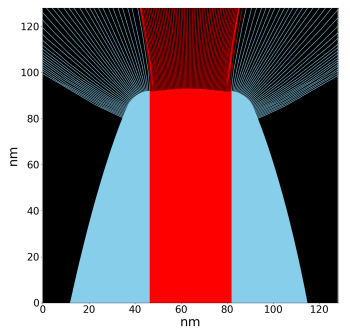

(d)

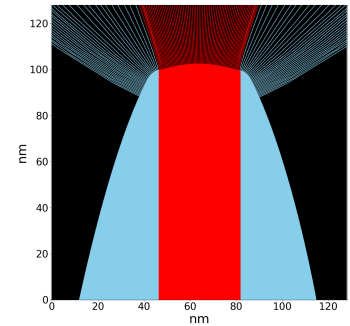

(b)

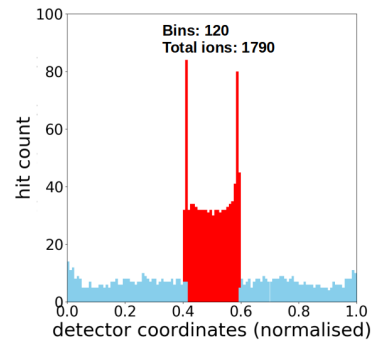

(e)

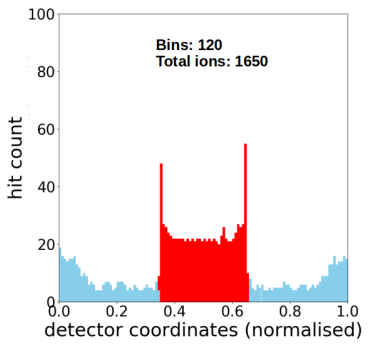

(c)

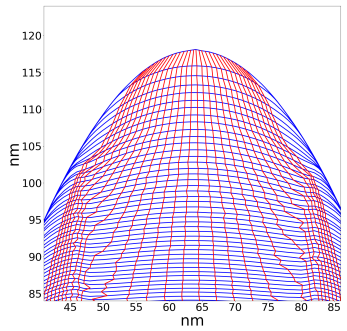

(f)

Figure 10: Simulation of the finFET-like geometry via the conductor continuum model assuming a lower evaporation field $\left(F_{0}\right.$ values of $30 \mathrm{Vnm}^{-1}$ and $\left.20 \mathrm{Vnm}^{-1}\right)$ for the central phase like in [6]. (a) Point-projection reconstruction of a finFETlike structure. (b) Partial simulated evaporation of the finFET-like structure and ion projection. (c) Corresponding 1D hitmap from ion projection at 9(b). (d) A later time in the same simulated evaporation and ion projection. (e) Corresponding 1D hitmap from ion projection at 9(d). (f) The reconstruction mapping for the simulated finFET-like evaporation. 


\subsection{Modelling Dielectrics}

When modelling dielectrics it is no longer valid to assume an equipotential sample surface. Instead the coupled FEM-BEM approach is required to correctly model the system electrostatics. In order to achieve this, the FEM-BEM scheme described in Section 3.3.4 was implemented.

\subsubsection{The Cylindrical Capacitor Test Geometry}

In order to verify the accuracy of the constant FEM-BEM coupling, the method was applied to a cylindrical capacitor test geometry. The test system consists of a FEM region, given by a central cylinder of fixed potential surrounded by an outer shell of floating potential, coupled to an exterior BEM region of infinite size (see Figure 11(a)). The exact value of the floating potential shell should depend on both the value of the fixed potential cylinder and the BEM boundary conditions at infinity. Due to the radial symmetry of the problem, it is possible to obtain an analytical solution and compare this with the numerical result. A visualisation of the potential field solution is shown in Figure 11(b) revealing the radial symmetry of the solution.

A cross-section of the numerical solution for particular mesh parameters (240 inputted boundary elements and 0.04 maximum element area) is compared to the analytical solution in Figure 11(c). Three individual factors govern the mesh construction performed by Triangle [52]: the input boundary mesh (both the external sample surface and internal phase boundaries via a $2 \mathrm{D}$ piecewise linear complex), the maximum triangle area, and the minimum internal angle allowed for triangle elements. Note that the mesh generated via Triangle can have additional boundary elements to that of the inputted boundary mesh. However, any additional boundary elements will only be generated through the subdivision of the inputted elements. Therefore any corners between elements present in the inputted boundary will remain within the constructed mesh.

By manual tuning it was found that the solution was largely insensitive to the minimum internal angle of elements, as long as its value was not to close to $0^{\circ}$ or $33^{\circ}$. A fixed minimum internal angle for mesh triangles of $\left(21^{\circ}\right)$ was imposed. The normalised error in the cross-sectional solution was then explored across a parameter subspace (see Figure 11(d)). As expected the parameter sweep revealed that the field converges to the analytical solution as the inputted boundary edge resolution increases and the maximum element size decreases. For the range of parameters explored, the parameter of particular importance in ensuring solution convergence is the input boundary. This is to be expected as increasing the inputted boundary mesh resolution (number of elements), reduces the angles between boundary element corners, such that the geometry converges towards the assumed 'smooth' form of the analytical solution.

In the implemented simulation framework, the input boundary mesh is set by the resolution of the level set grid and the contour extraction algorithm. 


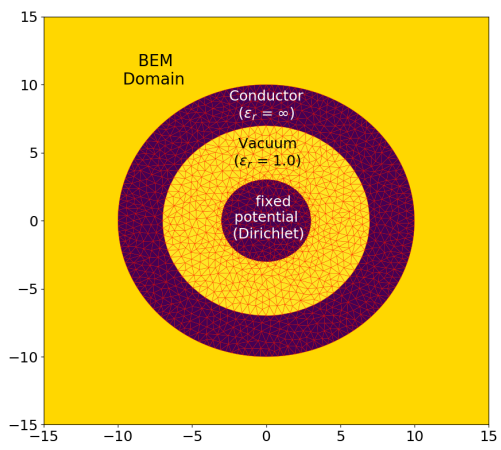

(a)

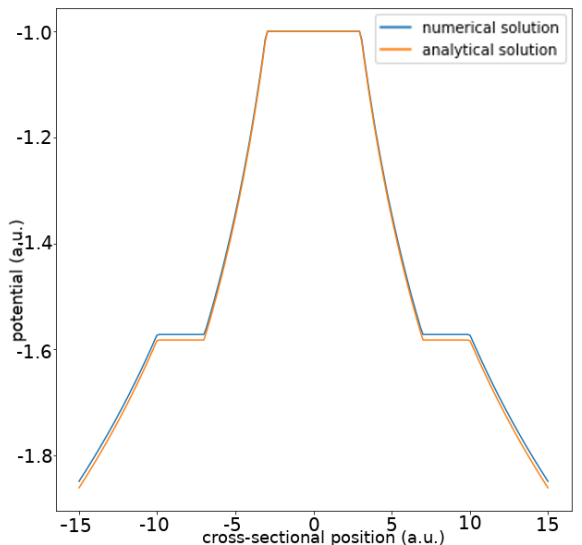

(c)

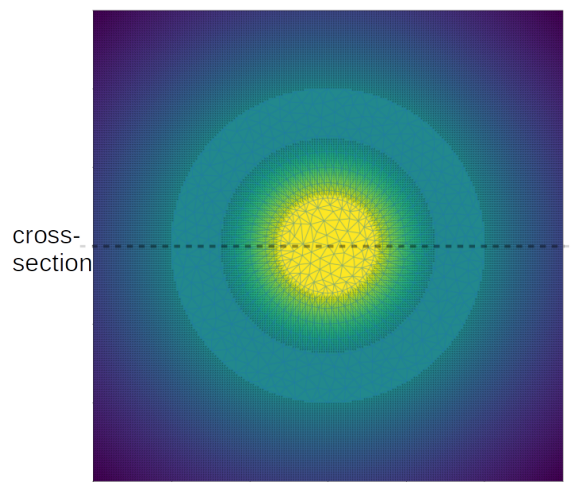

(b)

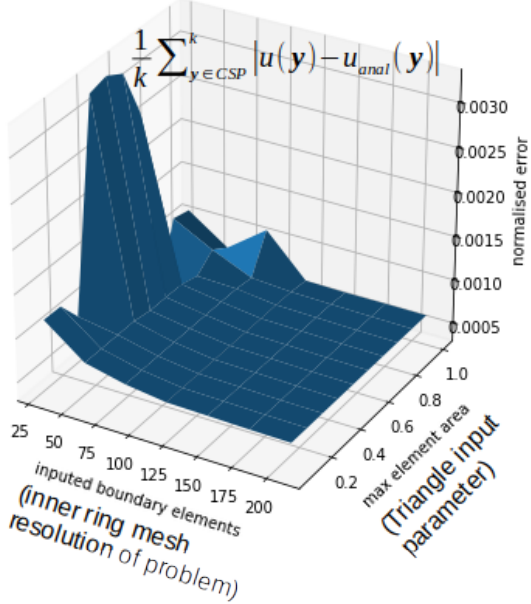

(d)

Figure 11: The cylindrical capacitor FEM-BEM test problem. (a) Setup for test problem. FEM mesh shown in red. Color indicates relative dielectric value. (b) Field solution for cylindrical capacitor test geometry. (c) Cross section of solution (numerical vs analytical) for 240 boundary elements. (d) Sweep across mesh generation parameter space (for fixed min internal element angle of $21^{\circ}$ ). The error formula sums over $k=120$ cross-sectional domain positions $\mathbf{y}$, where $u(\mathbf{y})$ is the FEM-calculated potential at $\mathbf{y}$ and $u_{a}(\mathbf{y})$ is the analytical potential. 


\subsubsection{Dielectric Effects and Trajectory Lensing}

The implemented FEM-BEM coupling scheme successfully reproduced similar field lensing and trajectory focusing effects for both a surface precipitate-type geometry (Figure 12) and a vertical layer geometry (Figure 13) to that produced by the finite difference model of Oberdorfer et. al. [32].

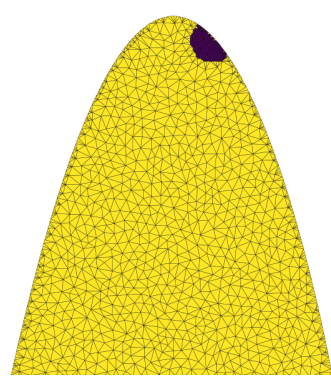

(a)

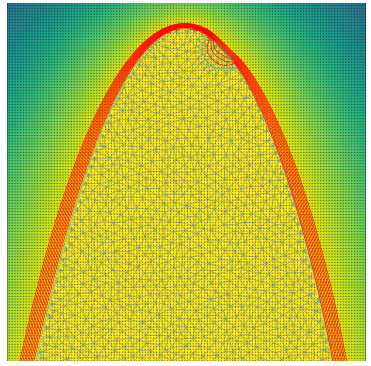

(b)

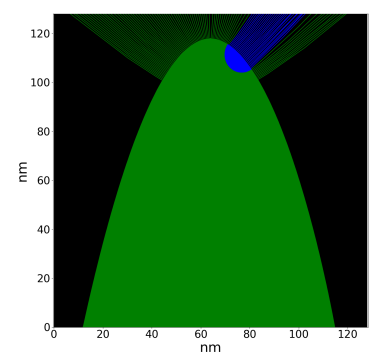

(c)

Figure 12: Simulation of a dielectric precipitate of relative permittivity $\epsilon_{r}=5$ surrounded by conducting material. Note that the lensing effect cannot be directly compared to that in three dimensions due to a missing dimension. (a) Generated FEM mesh for the surface precipitate geometry. (b) Lensing of field lines surrounding dielectric surface precipitate. (c) Resulting trajectory focusing due to field lensing.

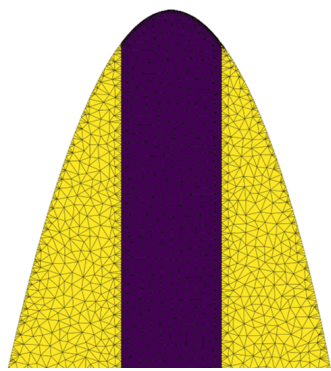

(a)

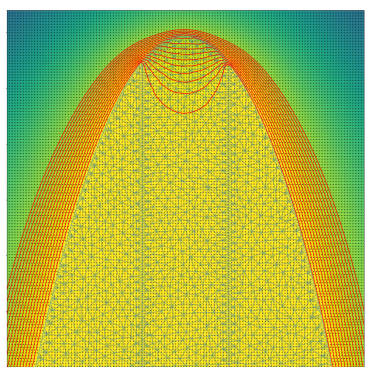

(b)

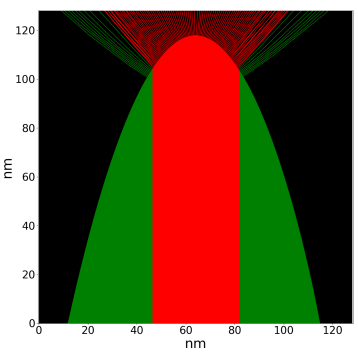

(c)

Figure 13: Simulation of a central dielectric vertical multilayer of relative permittivity $\epsilon_{r}=10$ surrounded by conducting material. Note that the lensing effect cannot be directly compared to that in three dimensions due to a missing dimension. (a) Generated FEM mesh for the finFET geometry. (b) Lensing of field lines surrounding dielectric precipitate. (c) Resulting trajectory focusing due to field lensing. 


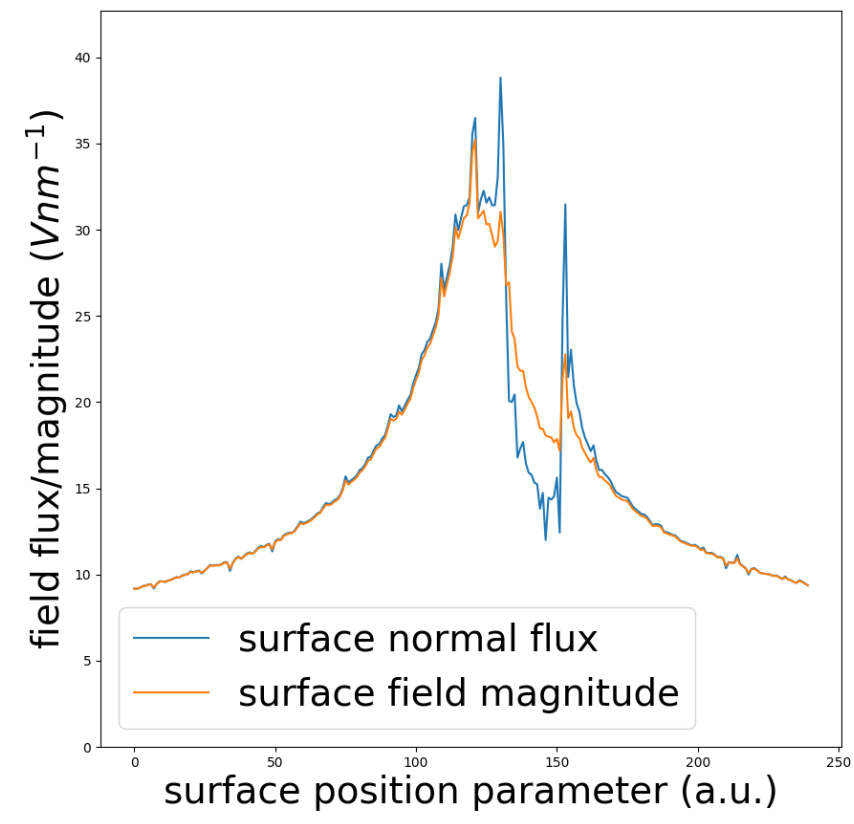

Figure 14: Plot of electric field surface normal component (blue), calculated via Eq. (10), and electric field strength (orange), calculated via Eq. (11), over the surface of the dielectric precipitate geometry (see Figure 12).

The finite difference model [32] showed that the potential lensing resulted in an enhancement of the sample surface electric field strength for both dielectric precipitates and layer geometries. This field enhancement contributed to an increased evaporation rate of dielectric material independent from the phase's evaporation field parameters. However, unlike Oberdorfer's model, the coupling scheme employed in this study failed to reproduce the same degree of field strength enhancement at the surface, with dielectric surface material instead resulting in a local reduction in the field strength. This failure can be seen in Figure 14, which plots the electric field surface normal component (blue) and field strength (orange) over the surface of the off-axis dielectric precipitate geometry shown in Figure 12. One possible explanation for this discrepancy is the reduced lensing effect present in two dimensions compared with three dimensions. Another issue could be the fixed potential panels of the constant BEM approximation failing to capture tangential field components close to the sample surface. Further testing is required to confirm the exact cause of this discrepancy. 


\subsection{Crystal Anisotropy}

A common artifact that appears erroneously within current reconstructions is the pole structure: low density regions arising due to sample faceting as a result of its underlying crystallographic structure. The level set method is capable of simulating such faceting behaviour by generalising the evaporation rate equation (1) to some anisotropic function. In this study this was achieved by generalising the evaporation field parameter $F_{0}$ in (1) to become some function of angle to the closest facet (see Section 3.4 for further details). The different phases in the simulation now have a set of associated facets associated, each with a given direction and magnitude. While numerous possibilities exist for simulating the evaporation of crystallographic materials systems, three case studies have been selected that we believe demonstrate its potential for simulating anisotropic systems while remaining simple to interpret.

The first case study considers the simulation of a homogeneous sample under an anisotropy function generated via Eq. (28). This anisotropy function, a radial plot of $F_{0}(\alpha)$ (see Section 3.4), is visualised in Figure 15(a). Simulated evaporation under this anisotropy function produced the faceted equilibrium form in Figure 15(c). The 2D analogue of the crystallographic Miller indices are labelled and compared between the faceted end-form and the visualised anisotropy function. Finally, the resulting 1D simulated hitmap is shown following ion projection off Figure 15(c). Two low density regions analogous to the pole structure seen on experimental detector hitmaps are produced corresponding to the facet corners between the $(10),(11)$, and $(-11)$ planes. While Figure 15(c) implies that two other low density regions should be present between both (11) and (-11), and the tip shank, these poles lie outside of the detector FOV. 


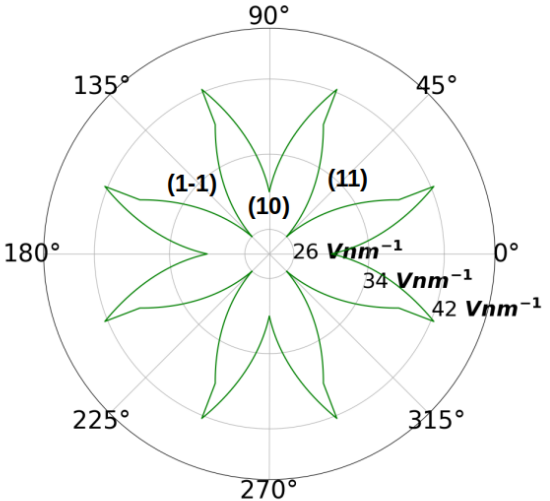

(a)

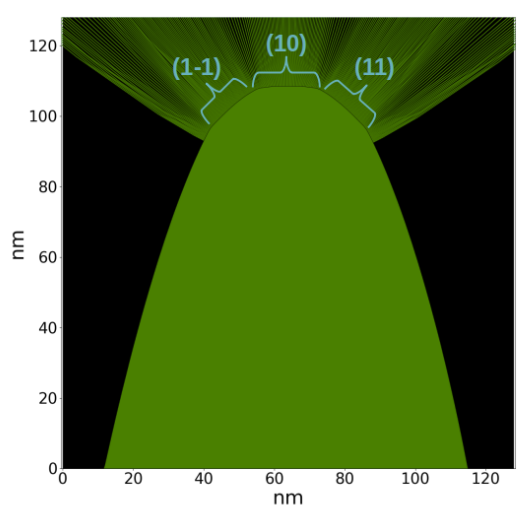

(c)

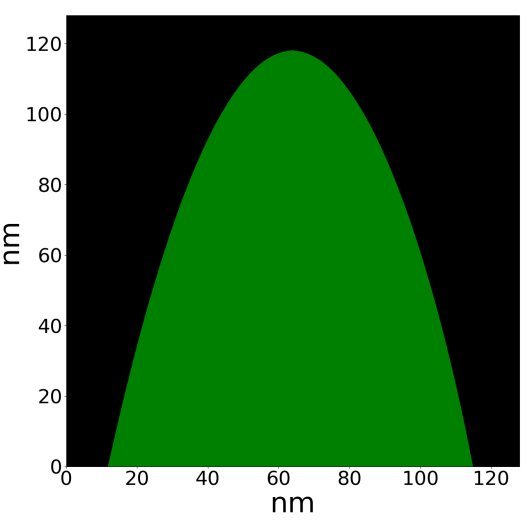

(b)

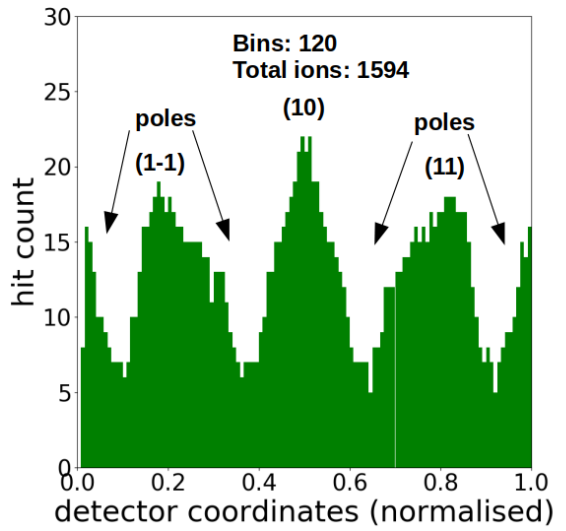

(d)

Figure 15: (a) Visualisation of an anisotropy function, generated via Eq. (28), with vertical, horizontal, and diagonal facets defined. $F_{0}$ values are $26 \mathrm{Vnm}^{-1}$ for (10)-type facets and $30 \mathrm{Vnm}^{-1}$ for (11)-type facets. (b) Initial unfaceted form prior to simulated evaporation. (c) Equilibrium form of homogeneous sample after simulated evaporation under the defined anisotropy function (see Figure 15(a)). (d) 1D hitmap following ion projection showing low density poles corresponding to facet corners.

The next case study considers the simulation of a vertical grain boundary (Figure 16). The two simulated crystal grains either side of the boundary have the same crystallographic structure but are misorientated at an angle of $22.5^{\circ}$. Such misorientation corresponds to a rotation in the anisotropy function. The anisotropy function for grain 2 is shown in Figure 16(a), while grain 1 is shown 
in Figure 16(b). Figure 16(c) contains a snapshot of the system after partial evaporation. Again the Miller indices corresponding to particular facets have been labelled and can be compared with the corresponding grain anisotropy functions.

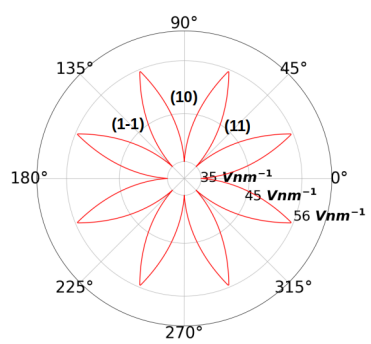

(a)

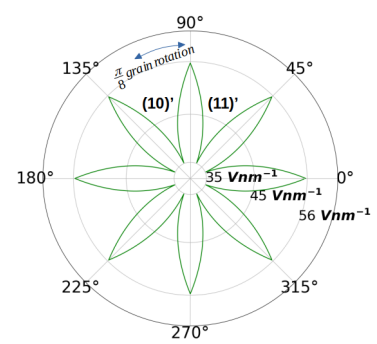

(b)

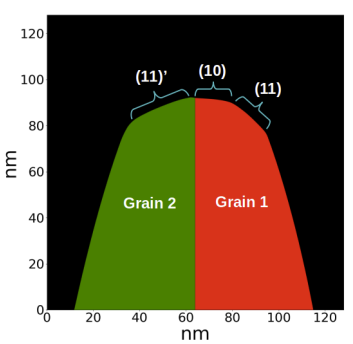

(c)

Figure 16: Simulated evaporation of vertical grain boundary with grain 2 at $22.5^{\circ}$ clockwise rotation to grain 1. (a) Anisotropy function for grain 1. (b) Anisotropy function for grain 2. (c) Simulated evaporation of a vertical grain boundary. $F_{0}$ values for all facets are $35 \mathrm{Vnm}^{-1}$.

The final case study considers the evaporation of a high evaporation field precipitate within an amorphous bulk. Frames of the simulation at different stages of evaporation are shown within Figure 17. Here the precipitate had a defined anisotropy function matching Figure 16(a). The precipitate faceting is most clear in Figure 17(c). Here a large flat top is clearly visible, corresponding to the (10) facet. Near the interface with the bulk the diagonal facets corresponding to (11) and (-11) can also be observed. Following evaporation of the precipitate, the remaining amorphous phase returns to its near hemispherical equilibrium form (see Figure 17(d)).

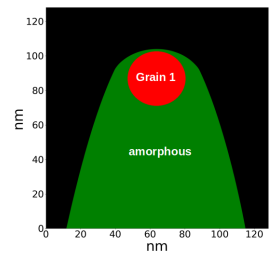

(a)

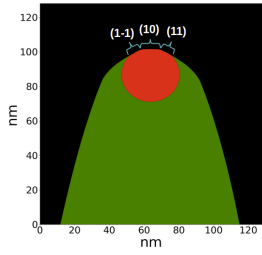

(b)

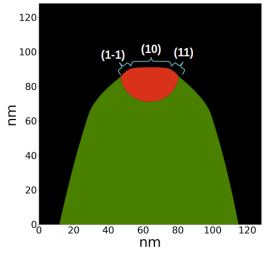

(c)

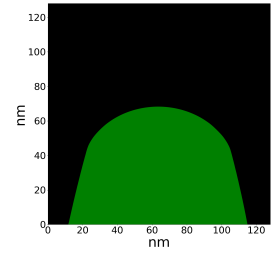

(d)

Figure 17: Simulated evaporation of a high evaporation field faceted precipitate (see Figure 16(a)) within an amorphous bulk of $F_{0}$ value $30 \mathrm{Vnm}^{-1}$. (a) Sample with precipitate prior to evaporation. (b) Partially evaporated precipitate. (c) Further evaporation of precipitate. evaporation.

(d) Equilibrium form after complete 


\subsection{Computational performance}

To demonstrate that the problem here remains highly tractable, which is critical to supporting a readily deployable reconstruction algorithm, we have undertaken several simple benchmarks. The current implementation features no explicit parallelism, and all simulations performed on a i7-4770 CPU $3.40 \mathrm{GHz}$ x 8 system. Until now all simulations included in this study have been run for a level set field grid of size $128 \times 128$. However, this grid resolution can be adjusted to any $N \times N$ system, assuming the input image for geometry initialisation has sufficient resolution for a smooth surface initialisation.

The time per iteration for the major algorithms are given in Figure 18(a) for increasing system size, with polynomials of the order of each component's theoretical asymptotic complexities being fitted. The naive closest-point extension velocity construction calculates the closest distance of each grid cell to the sample surface (where $N$ is the level set grid width and $k$ is the number of surface boundary elements), giving a time complexity in 2D of $O\left(N^{2} k\right) \sim O\left(N^{3}\right)$ and $O\left(N^{2}\right)$ memory complexity. The matrix inversion is performed via GaussJordan elimination with $O\left(k^{3}\right) \sim O\left(N^{3}\right)$ time complexity and $O\left(k^{3}\right) \sim O\left(N^{2}\right)$ memory complexity, while the BEM matrix construction has a time complexity in 2D of $O\left(k^{2}\right) \sim O\left(N^{2}\right)$ and $O\left(N^{2}\right)$ memory complexity.

This data reveals that the current bottleneck is the extension velocity construction. However, multiple options exist for accelerating this component including a narrowband grid implementation $[63,64,17]$ and a fast marching based extension velocity construction $[28,29]$. Combining both of these methods would reduce the theoretical time complexity to $O(N \log (N))$ and memory complexity to $O\left(N^{3}\right)$ in 2D. However, it remains unclear whether using such methods would improve the performance at the grid resolution used in this study.

The remaining two major components are the BEM matrix construction and inversion. While at the resolutions shown the matrix construction times appear to be increasing faster than the inversion step, extrapolating the fitted complexity plots reveals that the matrix inversion surpasses the matrix construction in the current implementation for spatial system sizes around $2000 \times 2000$ (not shown). Such a result implies that with further optimisation and parallelisation of the matrix construction, the matrix inversion step would be the theoretical bottleneck of the current algorithm. Note that on extension of the algorithm to three dimensions, these component complexities would scale differently (partially due to $k \sim N^{2}$ ).

The Figure 18(b) contains a normalised breakdown of the same components at grid sizes surrounding the resolution used within this study. At the study resolution of $128 \times 128$, the three components are of comparable fractions, with no particular component dominating. 


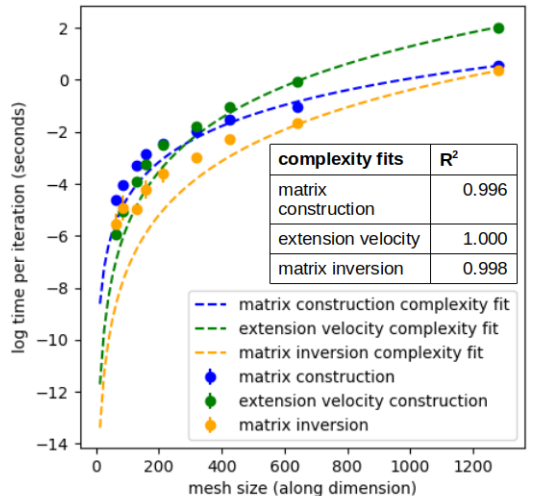

(a)

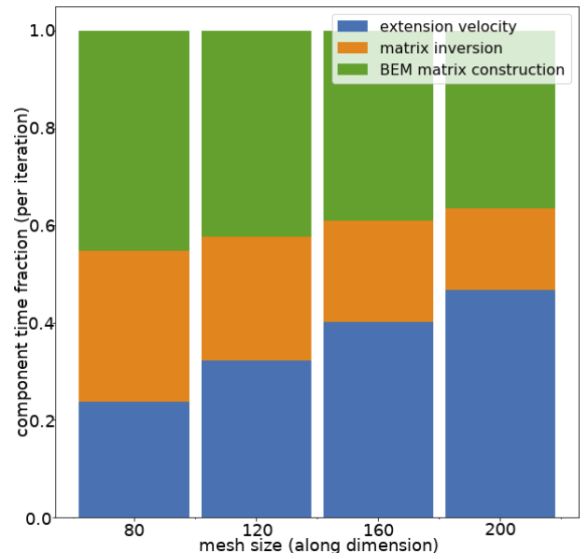

(b)

Figure 18: Performance metrics for the conductor BEM tip simulation algorithm. (a) Complexity plot for log time per algorithm iteration against system spatial resolution (level set grid). Theoretical complexity curves have also been fitted and their coefficient of determinations (strength of fit) given. (b) Breakdown of normalised algorithm major component times surrounding the grid resolution of $128 \times 128$ used within throughout this study.

The performance analysis above has only dealt with the surface modeling component of the algorithm, and has ignored the ion projection step. While currently this is the slowest component of the algorithm, this step is also highly parallelisable [65]. Theoretically, assuming a constant number of elements per panel, the theoretical time complexity should go as $O\left(k^{2}\right) \sim O\left(N^{2}\right)$ (assuming a collocation BEM) and memory complexity $O(N)$, as the sample-detector distance is independent from the level set grid dimension $N$.

Finally, it is worth briefly considering FEM-BEM coupling extension for handling dielectrics. Due to the internal mesh, the time complexity of solving the system via LU decomposition is $O\left(k^{3}\right) \sim O\left(N^{6}\right)$ and memory complexity $O\left(N^{4}\right)$ (when storing as a dense matrix). However, at the grid resolution of $128 \times 128$ the time for total sample evaporation remains significantly faster than atomic models. The Figure 19 shows a particular simulation for a low evaporation field dielectric precipitate of $F_{0}=20 \mathrm{Vnm}^{-1}$ and a dielectric constant of $\epsilon_{r}=30$, embedded within a conductor bulk of $F_{0}=30 \mathrm{Vnm}^{-1}$. 


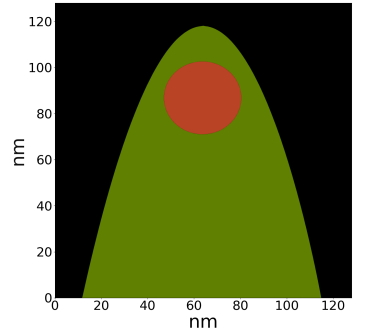

(a)

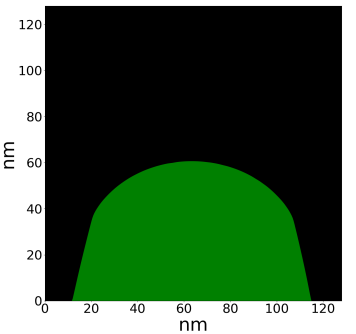

(b)

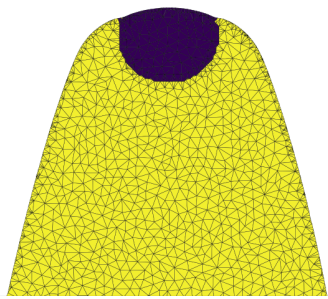

(c)

Figure 19: Geometry setup for dielectric simulation test. Simulated geometry consists of an on-axis precipitate $\left(F_{0}\right.$ values of $30 \mathrm{Vnm}^{-1}$ and $20 \mathrm{Vnm}^{-1}$, and a dielectric constant of $\epsilon_{r}=30$ ). (a) Initial geometry for dielectric simulation (onaxis precipitate). (b) Final geomery of sample at end of collapse. (c) Typical internal mesh constructed throughout collapse (dependent on mesh parameters).

Three different running modes were compared for this geometry: a complete FEM-BEM simulation throughout the entire collapse, a pure BEM conductor simulation ignoring dielectric behaviour, and a hybrid mode whereby the FEMBEM coupling mode was only executed on the dielectric precipitate intersecting the sample surface. The evaporation times for each mode are summarised in Table 1. It is worth noting that these evaporation times are geometry dependent and that changing various parameters (e.g. FEM mesh resolution, CFL time fraction) can significantly modify collapse times. Ion projection and graphical outputs were disabled throughout these simulations.

\begin{tabular}{||cc||}
\hline Method & Simulation times (seconds) \\
\hline \hline FEM-BEM & 670 \\
Pure BEM & 100 \\
hybrid & 470 \\
\hline
\end{tabular}

Table 1: Results for collapse of test dielectric geometry (see Figure 19) under three different modes. Results averaged over 3 runs (with 3 runs prior discarded to maximise disk caching) to average out operating system jitter. Reported times rounded to nearest 10 seconds due to precision of Python's time.time() method. 


\section{Limitations and Improvements}

The major limitation of this current model for predicting experimental field evaporation behaviour is the fact that it is two dimensional. The electric field behaves differently in three dimensions, limiting the direct applicability of the model to making predictions regarding experimental evaporation phenomena. This limitation can be clearly seen by the discrepancy in the predicted evaporation behaviour for dielectrics compared with previous electrostatic simulation work [32]. Nevertheless, extending the current model to three dimensions is mainly an implementation exercise. Thus we believe that despite our results here being presented in two dimensions, this approach will be readily extendable to three dimensions. However, the additional dimension will cause a significant increase in computational overhead. Further optimization techniques will be required in order to maintain the computational performance.

Currently some of the slowest components are the BEM and FEM-BEM matrix inversion, and the ion projection step, which is also restricted by the efficiency of the BEM field calculations. Existing methods for accelerating the BEM boundary calculation step include the fast multipole method [37, 66], adaptive cross-approximation [66], and pre-corrected fast fourier transform methods [66]. The fast multipole BEM could also be used to potentially accelerate ion trajectory calculations via calculation of external domain field points, although an octree structure would have to be constructed over this space [67]. Iterative methods to solving the FEM-BEM system could further accelerate the computation [68] by allowing the use of specialised solvers for the separate FEM and BEM components such as the conjugate gradient method and GMRES.

The number of required ion trajectory integrations for constructing the reconstruction mapping could be further reduced through a more intelligent trajectory sampling: adapting the ion projection density and frequency depending on local changes in surface geometry. Ion trajectories could also be extrapolated by analytical functions after a sufficient distance from the sample surface, eliminating the need for complete projection up to the detector. The best course of optimisation remains unclear at this time, although should become clearer on extension to 3D and with implementation of a prototype reconstruction algorithm.

It is noted that numerical issues exist with the FEM-BEM coupling. The constant BEM approximates elements as being of constant potential. This approximation fails to reproduce tangential fluxes close to the surface and complicates the coupling procedure with the linear FEM where nodes lie at element corners. This breakdown in the representation of the tangental field close to surface could be partially responsible for the discrepancy in dielectric evaporation behaviour from alternative electrostatic models [32]. However, it is difficult to draw conclusions due to the discrepancy in the dimensionality. A multidomain BEM implementation would likely prove more efficient than the FEM-BEM coupling [69, 70]. However, such a method would be unable to account for spatially non-linear parameters, which will likely become relevant 
when attempting to model the effect of the laser on sample evaporation.

The accuracy of the pure BEM and FEM-BEM calculations could be further increased by using a Galerkin BEM $[71,72,58]$, which can also naturally handle issues arising from boundary mesh corners. However, using such Galerkin BEM formulations comes with an increased computational overhead.

Multiple approaches exist for potentially accelerating the level set component of the algorithm. Currently this grid employs a full-band method, unnecessarily simulating grid points far away from the interface. Previously we have demonstrated a narrowband implementation where only grid points sufficiently close to the interface are tracked $[63,17]$. This narrowband of active field points is then updated as the embedded sample surface propagates. Finally, further acceleration could be achieved via parallelisation of the level set algorithm, either by distribution of the level set grid nodes across multiple cores [73] or through a parallel-in-time integration scheme [74].

While the effect of the laser on sample evaporation has been omitted from this study, it is known to have a major effect on sample evaporation [9]. A simplified model could be implemented by generalising the temperature parameter in equation (1) such that it varies with lateral position over the sample surface corresponding to the laser flux asymmetry $[75,76]$.

\section{Conclusion}

The current projection approach to APT reconstruction introduces major distortions into multiphase material systems by approximating the sample apex geometry as a hemispherical cap. There is numerous experimental evidence that such simple geometric approximations breakdown for more complicated sample structures $[8,21,9,10]$. In order to correct for these distortions a new reconstruction protocol is required that accounts for the changing sample geometry during field evaporation.

Unlike the traditional atomic model approach, the developed level set model presented here considers the sample as a continuum. The model accurately solves the electric field at the sample surface through the Boundary Element Method (BEM) and a FEM-BEM coupling without having to extend a mesh into the vacuum. This framework also allows for the inclusion of crystallographic anisotropy. Such an approach builds upon previous work using a curvature flow approximation for the electric field $[16,17]$ and grid-based finite difference electric field solvers [33]. By modelling the sample as a continuum and ignoring the underlying crystallographic structure, sample evaporation is simulated in minutes (see Table 1) as opposed to the hours-to-days required by atomicresolution electrostatic models.

While the current implementation is only two-dimensional, nothing immediately obvious is preventing the outlined method from being extended to three-dimensions. When calibrated with experiment, such a three-dimensional model could be harnessed to obtain an ion trajectory mapping between the detector and sample surface which could be applied to driving a new distortion 
correcting APT reconstruction algorithm.

\section{Acknowledgements}

Charles Fletcher acknowledges support from CAMECA ${ }^{\circledR}$ for financially supporting this research. Michael P. Moody and Daniel Haley acknowledge support from the ESPRC project Hems EP/L014742/1. We would also like to thank Claudia Fleischmann of IMEC for sharing with us their finFET-like APT

dataset, originally published in [10], as well as Benjamin Jenkins for providing the SEM sample image.

\section{Source Code}

The source code for the 2D simulation tool CASRA (Continuum APT Simulation and Reconstruction Aid) used throughout this study can be accessed and downloaded from https://gitlab.com/fletchie/casra. All simulations in this study were performed on Ubuntu 18.04, with Python libraries installed via pip3 and linked against OpenBLAS. Specific parameter configurations for simulations can be found in the repository examples. 


\section{References}

[1] P. Bas, A. Bostel, B. Deconihout, and D. Blavette. A general protocol for the reconstruction of 3D atom probe data. Applied Surface Science, 87/88:298-304, 1995.

[2] B. P. Geiser, D. J. Larson, E. Oltman, S. S. A. Gerstl, D. A. Reinhard, T. F. Kelly, and T. J. Prosa. Wide-Field-of-View Atom Probe Reconstruction. Microscopy and Microanalysis, 15(Suppl 2):292-293, 2009.

[3] B. Gault, D. Haley, F. De Geuser, M. P. Moody, E. A. Marquis, D. J. Larson, and B. P. Geiser. Advances in the reconstruction of atom probe tomography data. Ultramicroscopy, 111(6):448-457, 2011.

[4] F. De Geuser and B. Gault. Reflections on the Projection of Ions in Atom Probe Tomography. Microscopy and Microanalysis, 23(2):238-246, 2017.

[5] A. Cerezo, L. Chang, P. Clifton, M. Galtrey, S. Gerstl, C. Humphreys, M. Mueller, R. Oliver, G. Smith, and Y. Wu. 3D Atom Probe Analysis of Quantum Well and Quantum Dot Materials. Microscopy and Microanalysis, 13(S02):1608-1609, 2007.

[6] D. Melkonyan, C. Fleischmann, L. Arnoldi, J. Demeulemeester, A. Kumar, J. Bogdanowicz, F. Vurpillot, and W. Vandervorst. Atom probe tomography analysis of SiGe fins embedded in $\mathrm{SiO} 2$ : Facts and artefacts. Ultramicroscopy, 179:100-107, 2017.

[7] B. Langelier, X. Wang, and K. Grandfield. Atomic scale chemical tomography of human bone. Scientific Reports, 7:1-9, 2017.

[8] E. A. Marquis and F. Vurpillot. Chromatic aberrations in the field evaporation behavior of small precipitates. Microscopy and Microanalysis, 14(6):561-570, 2008.

[9] S. Koelling, N. Innocenti, A. Schulze, M. Gilbert, A. K. Kambham, and W. Vandervorst. In-situ observation of non-hemispherical tip shape formation during laser-assisted atom probe tomography. Journal of Applied Physics, 109(10), 2011.

[10] D. Melkonyan, C. Fleischmann, L. Arnoldi, J. Demeulemeester, A. Kumar, J. Bogdanowicz, F. Vurpillot, and W. Vandervorst. Atom probe tomography analysis of $\mathrm{SiGe}$ fins embedded in $\mathrm{SiO} 2$ : Facts and artefacts. Ultramicroscopy, 179:100-107, 2017.

[11] D. Beinke, C. Oberdorfer, and G. Schmitz. Towards an accurate volume reconstruction in atom probe tomography. Ultramicroscopy, 165:34-41, 2016. 
[12] N. Rolland, D. J. Larson, B. P. Geiser, S. Duguay, F. Vurpillot, and D. Blavette. An analytical model accounting for tip shape evolution during atom probe analysis of heterogeneous materials. Ultramicroscopy, 159:195$201,2015$.

[13] N. Rolland, F. Vurpillot, S. Duguay, B. Mazumder, J. S. Speck, and D. Blavette. New Atom Probe Tomography Reconstruction Algorithm for Multilayered Samples: Beyond the Hemispherical Constraint. Microscopy and Microanalysis, 23(2):247-254, 2017.

[14] B. Gault, S. T. Loi, V. J. Araullo-Peters, L. T. Stephenson, M. P. Moody, S. L. Shrestha, R. K. W. Marceau, L. Yao, J. M. Cairney, and S. P. Ringer. Dynamic reconstruction for atom probe tomography. Ultramicroscopy, 111(11):1619-1624, 2011.

[15] J. A. Sethian. Level Set Methods and Fast Marching Methods: Evolving Interfaces in Computational Geometry, Computer Vision and Materials Science. Cambridge University Press, 2nd edition, 1999.

[16] D. Haley, M. P. Moody, and G. D. W. Smith. Level set methods for modelling field evaporation in atom probe. Microscopy and Microanalysis, 19(6):1709-17, 2013.

[17] D. Haley, P. A. J. Bagot, and M. P. Moody. Extending continuum models for atom probe simulation. Materials Characterization, 146:299-306, 2018.

[18] K. X. H. Zhao, H. Power, and L. C. Wrobel. Numerical simulation of dendritic crystal growth in a channel. Engineering Analysis with Boundary Elements, 19(4):331-337, 1997.

[19] T. T. Tsong. Field Ion Emission. In Atom-Probe Field Ion Microscopy, chapter 2, pages 10-102. Cambridge University Press, 1 edition, 1990.

[20] D. Haley, T. C. Petersen, S. P. Ringer, and G. D. W. Smith. Atom probe trajectory mapping using experimental tip shape measurements. Journal of Microscopy, 244(2):170-180, 2011.

[21] E. A. Marquis, B. P. Geiser, T. J. Prosa, and D. J. Larson. Evolution of tip shape during field evaporation of complex multilayer structures. Journal of Microscopy, 241:225-233, 2011.

[22] S. Du, T. Burgess, S. Tjing Loi, B. Gault, Q. Gao, P. Bao, L. Li, X. Cui, W. Kong Yeoh, H. Hoe Tan, C. Jagadish, S. P. Ringer, and R. Zheng. Full tip imaging in atom probe tomography. Ultramicroscopy, 124:96-101, 2013.

[23] C. A. Brebbia, J. C. F. Telles, and L. C. Wrobel. Potential Problems. In Boundary Element Techniques: Theory and Applications in Engineering, chapter 2, pages 47-108. Springer-Verlag, Heidelberg, 1 edition, 1984. 
[24] M. K. Miller and R. Forbes. Chapter 3: Field Evaporation and Related Topics. In Atom-Probe Tomography: The Local Electrode Atom Probe, chapter 3, pages 111-187. Springer, 2014.

[25] R. Forbes. Field evaporation theory: a review of basic ideas. Applied Surface Science, 87-88(94):1-11, 1995.

[26] J. A. Sethian and J. Strain. Crystal Growth and Dendritic Solidification. Journal for Computational Physics, 98:231-253, 1992.

[27] R. Malladi, J. A. Sethian, and B. C. Vemuri. Shape modeling with front propagation: a level set approach. 17(2):158-175, 1995.

[28] D. Adalsteinsson and J. A. Sethian. The fast Construction of extension Velocities in Level Set Methods. The Journal of Computational Physics, 148:2-22, 1999.

[29] D. L. Chopp. Another Look at Velocity Extensions in the Level Set Method. SIAM Journal on Scientific Computing, 31(5):3255-3273, 2009.

[30] C. Oberdorfer, S. M. Eich, and G. Schmitz. A full-scale simulation approach for atom probe tomography. Ultramicroscopy, 128:55-67, 2013.

[31] F. Vurpillot, A. Cerezo, D. Blavette, and D. J. Larson. Modeling Image Distortions in 3DAP. Microscopy and microanalysis, 10(3):384-390, 2004.

[32] C. Oberdorfer and G. Schmitz. On the field evaporation behavior of dielectric materials in three-dimensional atom probe: A numeric simulation. Microscopy and Microanalysis, 17(1):15-25, 2011.

[33] Z. Xu, D. Li, W. Xu, A. Devaraj, R. Colby, S. Thevuthasan, B. P. Geiser, and D. J. Larson. Simulation of heterogeneous atom probe tip shapes evolution during field evaporation using a level set method and different evaporation models. Computer Physics Communications, 189:106-113, 2015 .

[34] J. A. Sethian. Coupling to Applications: The Fast Construction of Extension Velocities. In Level Set Methods and Fast Marching Methods, chapter 11, pages 127-140. Cambridge University Press, 2 edition, 1999.

[35] N. Rolland, F. Vurpillot, S. Duguay, and D. Blavette. A Meshless Algorithm to Model Field Evaporation in Atom Probe Tomography. Microscopy and Microanalysis, 21(6):1649-1656, 2015.

[36] S. Tjing, B. Gault, S. P. Ringer, D. J. Larson, and B. P. Geiser. Electrostatic simulations of a local electrode atom probe : The dependence of tomographic reconstruction parameters on specimen and microscope geometry. Ultramicroscopy, 132:107-113, 2013. 
[37] L. Yijun. Fast Multipole Boundary Element Method: Theory and Applications in Engineering. Cambridge University Press, Cambridge, 1st edition, 2009.

[38] M. G. Larson and F. Bengzon. The Finite Element Method in 2D. In The Finite Element Method: Theory, Implementation, and Applications, chapter 4, pages 71-111. Springer, 2013.

[39] F. Vurpillot, A Gaillard, G. Da Costa, and B. Deconihout. Ultramicroscopy A model to predict image formation in Atom probe Tomography. Ultramicroscopy, 132:152-157, 2013.

[40] E. P. Silaeva, L. Arnoldi, M. L. Karahka, B. Deconihout, A. Menand, H. J. Kreuzer, and A. Vella. Do dielectric nanostructures turn metallic in highelectric dc fields? Nano Letters, 14(11):6066-6072, 2014.

[41] L. Arnoldi, E. P. Silaeva, A. Gaillard, F. Vurpillot, I. Blum, L. Rigutti, B. Deconihout, and A. Vella. Energy deficit of pulsed-laser field-ionized and field-emitted ions from non-metallic nano-tips. Journal of Applied Physics, 115(20), 2014.

[42] L. Arnoldi, E. P. Silaeva, F. Vurpillot, B. Deconihout, E. Cadel, I. Blum, and A. Vella. Role of the resistivity of insulating field emitters on the energy of field-ionised and field-evaporated atoms. Ultramicroscopy, 159:139-146, 2015 .

[43] C. Oberdorfer, S. M. Eich, M. Lütkemeyer, and G. Schmitz. Applications of a versatile modelling approach to $3 \mathrm{D}$ atom probe simulations. Ultramicroscopy, 159:184-194, 2015.

[44] M. Burger, F. Haußer, C. Stöcker, and A. Voigt. A level set approach to anisotropic flows with curvature regularization. Journal of Computational Physics, 225(1):183-205, 2007.

[45] Y. Giga and N. Požár. A level set crystalline mean curvature flow of surfaces. Adv. Differential Equations, 21(7-8):631-698, 2016.

[46] B. T. Murray, A. A. Wheeler, and M. E. Glicksman. Simulations of experimentally observed dendritic growth behavior using a phase-field model. Journal of Crystal Growth, 154(3-4):386-400, 1995.

[47] G. Russo and P. Smereka. A level-set method for the Evolution of Faceted Crystals. SIAM Journal on Scientific Computing, 21(6):2073-2095, 2000.

[48] F. Vurpillot, A. Bostel, and D. Blavette. The shape of field emitters and the ion trajectories in three-dimensional atom probes. Journal of Microscopy, 196(December):332-336, 1999.

[49] T.L Einstein. Equilibrium Shape of Crystals, volume 1. 2014. 
[50] B. Gault, M. P. Moody, F. De Geuser, and A. La Fontaine. Spatial Resolution in Atom Probe Tomography M icroscopy M icroanalysis Spatial Resolution in Atom Probe Tomography. Microscopy and Microanalysis, 16:99-110, 2010.

[51] W. E. Lorensen and H. E. Cline. Marching cubes: A high resolution 3D surface construction algorithm. Computer Graphics, 21(4):163-169, 1987.

[52] J. R. Shewchuk. Triangle: Engineering a 2D quality mesh generator and Delaunay triangulator. In 1st Workshop on Applied Computational Geometry, pages 203-222, 1996.

[53] J. R. Dormand and P. J. Prince. A reconsideration of some embedded Runge-Kutta formulae. Journal of Computational and Applied Mathematics, 15(2):203-211, 1986.

[54] F. Vurpillot, A. Bostel, and D. Blavette. Trajectory overlaps and local magnification in three-dimensional atom probe. Applied Physics Letters, $76(21): 3127-3129,2000$.

[55] J. Friedrich. A linear analytical boundary element method (BEM) for 2D homogeneous potential problems. Computers and Geosciences, 28(5):679692, 2002.

[56] C. A. Brebbia, J. C. F. Telles, and L. C. Wrobel. Interpolation Functions. In 1Boundary Element Techniques: Theory and Applications in Engineering. Springer-Verlag, Heidelberg, 1 edition, 1984.

[57] L. J. Gray and G. H. Paulino. Symmetric galerkin boundary integral formulation for interface and multi-zone problems. International Journal for Numerical Methods in Engineering, 40(16):3085-3101, 1997.

[58] A. Sutradhar, G. H. Paulino, and L. J. Gray. Symmetric Galerkin Boundary Element Method. Springer, Heidelberg, 1 edition, 2008.

[59] A. Grenier, S. Duguay, J. P. Barnes, R. Serra, G. Haberfehlner, D. Cooper, F. Bertin, S. Barraud, G. Audoit, L. Arnoldi, E. Cadel, A. Chabli, and F. Vurpillot. 3D analysis of advanced nano-devices using electron and atom probe tomography. Ultramicroscopy, 136:185-192, 2014.

[60] A. Grenier, S. Duguay, J. P. Barnes, R. Serra, N. Rolland, G. Audoit, P. Morin, P. Gouraud, D. Cooper, D. Blavette, and F. Vurpillot. Three dimensional imaging and analysis of a single nano-device at the ultimate scale using correlative microscopy techniques. Applied Physics Letters, 106(21), 2015.

[61] J. P. Barnes, A. Grenier, I. Mouton, S. Barraud, G. Audoit, J. Bogdanowicz, C. Fleischmann, D. Melkonyan, W. Vandervorst, S. Duguay, N. Rolland, F. Vurpillot, and D. Blavette. Atom probe tomography for advanced nanoelectronic devices: Current status and perspectives. Scripta Materialia, 148:91-97, 2017. 
[62] F. Vurpillot, N. Rolland, R. Estivill, S. Duguay, and D. Blavette. Accuracy of analyses of microelectronics nanostructures in atom probe tomography. Semiconductor Science and Technology, 31(7), 2016.

[63] D. L. Chopp. Computing Minimal Surfaces via Level Set Curvature Flow. Journal for Computational Physics, 106:77-91, 1992.

[64] D. Adalsteinsson and J. A. Sethian. A Fast Level Set Method for Propagating Interfaces. Journal for Computational Physics, 118:267-277, 1994.

[65] N. V. Miluchihin, K. Miura, and M. Inoue. Application of a Parallel Computer to Simulation of Ion Trajectories in an Ion Cyclotron Resonance Spectrometer. Rapid Communications In Mass Spectrometry, 7:966-970, 1993.

[66] Y. J. Liu, S. Mukherjee, N. Nishimura, M. Schanz, W. Ye, A. Sutradhar, E. Pan, N. A. Dumont, A. Frangi, and A. Saez. Recent Advances and Emerging Applications of the Boundary Element Method. Applied Mechanics Reviews, 64(3):030802, 2012.

[67] J. Ptaszny and F. Fedelinski. Stress analysis of linear elastic structures by the fast multipole boundary element method. Computer Assisted Mechanics and Engineering Sciences, 16(3-4):223-240, 2009.

[68] G. Aiello, S. Alfonzetti, E. Dilettoso, and N. Salerno. An iterative solution to FEM-BEM algebraic systems for open-boundary electrostatic problems. IEEE Transactions on Magnetics, 43(4):1249-1252, 2007.

[69] X. W. Gao, L. Guo, and C. Zhang. Three-step multi-domain BEM solver for nonhomogeneous material problems. Engineering Analysis with Boundary Elements, 31(12):965-973, 2007.

[70] M. Ramšak and L. Škerget. 3D multidomain BEM for solving the Laplace equation. Engineering Analysis with Boundary Elements, 31(6):528-538, 2007.

[71] M. Aurada, M. Feischl, T. Führer, M. Karkulik, J. M. Melenk, and D. Praetorius. Classical FEM-BEM coupling methods: Nonlinearities, wellposedness, and adaptivity. Computational Mechanics, 51(4):399-419, 2013.

[72] R. Springhetti, G. Novati, and M. Margonari. Weak coupling of the symmetric galerkin BEM with FEM for potential and elastostatic problems. CMES - Computer Modeling in Engineering and Sciences, 13(1):67-80, 2006.

[73] M. Mirzadeh, A. Guittet, C. Burstedde, and F. Gibou. Parallel level-set methods on adaptive tree-based grids. Journal of Computational Physics, 322:345-364, 2016. 
[74] D. Ruprecht and R. Krause. Explicit parallel-in-time integration of a linear acoustic-advection system. Computers and Fluids, 59:72-83, 2012.

[75] G. Sha, A. Cerezo, and G. D. W. Smith. Field evaporation behavior during irradiation with picosecond laser pulses. Applied Physics Letters, 92(4):2006-2009, 2008.

[76] J. Houard, A. Vella, F. Vurpillot, and B. Deconihout. Three-dimensional thermal response of a metal subwavelength tip under femtosecond laser illumination. Physical Review B - Condensed Matter and Materials Physics, 84(3):1-4, 2011. 